Electronic version of an article published as International Journal of Wavelets, Multiresolution and Information Processing Vol. 6, No. 2 (2008) 249-277.

DOI: $10.1142 / S 0219691308002331$.

Copyright World Scientific Publishing Company, www.worldscinet.com/ijwmip.

\title{
PERTURBATION TECHNIQUES IN IRREGULAR SPLINE-TYPE SPACES
}

\author{
HANS G. FEICHTINGER, URSULA MOLTER, AND JOSÉ LUIS ROMERO
}

\begin{abstract}
In this article we study various perturbation techniques in the context of irregular spline-type spaces. We first present the sampling problem in this general setting and prove a general result on the possibility of perturbing sampling sets. This result can be regarded as an spline-type space analogue in the spirit of Kadec's Theorem for bandlimited functions (see [14] and [15]). We further derive some quantitative estimates on the amount by which a sampling set can be perturbed, and finally prove a result on the existence of optimal perturbations (with the stability of reconstruction being the optimality criterion). Finally, the techniques developed in the earlier parts of the article are used to study the problem of disturbing a basis for a spline-type space, in order to derive a sufficient criterion for a space generated by irregular translations to be a spline-type space.
\end{abstract}

\section{Preliminaries And notation}

1.1. Notation. We will work with functions on $\mathbb{R}^{d}$, and denote the Euclidean norm of $x \in \mathbb{R}^{d}$ by $|x|_{2}$, and maximum norm by $|x|_{\infty}=\max _{1 \leq k \leq d}\left|x_{k}\right|$.

Definition 1.1. For a complex valued function $f: \mathbb{R}^{d} \rightarrow \mathbb{C}$ and $M \subseteq \mathbb{R}^{d}$, we denote by $\sup _{M}|f|$ the supremum of the absolute values of $f$ on $M$, i.e.

$$
\sup _{M}|f|:=\sup \{|f(x)|: x \in M\} .
$$

For $\delta>0$ and $x \in \mathbb{R}^{d}$, we define the $\delta$-oscillation of $f$ at $x$ by

$$
\operatorname{osc}_{\delta}(f)(x):=\sup \left\{|f(x)-f(y)|:|y-x|_{2} \leq \delta\right\} .
$$

\section{2. p-Riesz bases.}

Definition 1.2. Let $1 \leq p<\infty, B$ a Banach space and $\Lambda$ a countable index set. We say that a family $\left\{f_{k}\right\}_{k \in \Lambda}$ in $B$ is a p-Riesz basis for $B$ if the map

$$
\begin{aligned}
& \ell^{p}(\Lambda) \longrightarrow B \\
& \left(c_{k}\right)_{k} \mapsto \sum_{k \in \Lambda} c_{k} f_{k},
\end{aligned}
$$

is well defined and continuously invertible.

Since $B$ is a Banach space, it suffices that the map is a continuous linear isomorphism because Banach's theorem implies continuity of the inverse mapping.

We will call the family $\left\{f_{k}\right\}_{k \in \Lambda}$ a $p$-Riesz sequence if it is a p-Riesz basis of its closed linear span. That is, if the operator in (1) is both bounded above and below.

\footnotetext{
Date: April 6, 2008.

${ }^{1}$ Under this assumption the series converges unconditionally in $B$, because the "finite" sequences are dense in $B$.
} 


\subsection{Separation of point sets.}

Definition 1.3. Let $X \equiv\left\{x_{k}\right\}_{k \in \Lambda}$ in $\mathbb{R}^{d}$ be an indexed family of points, where the indexing set $\Lambda$ is countable. The separation of $X$ is defined by

$$
\operatorname{sep}(X):=\inf _{k \neq j}\left|x_{k}-x_{j}\right|_{\infty}
$$

and we say that $X$ is uniformly separated if $\operatorname{sep}(X)>0$.

For each $\alpha>0$, we define the relative separation of order $\alpha$ of $X$ by

$$
r e l_{\alpha}(X):=\sup _{b \in \mathbb{R}^{d}} \#\left\{k \in \Lambda: x_{k} \in[-\alpha, \alpha)^{d}+b\right\},
$$

and say that $X$ is relatively separated if $\operatorname{rel}_{\alpha}(X)<\infty$, for same value of $\alpha>0$.

For technical reasons we will use occasionally the following variant. Let us define the strong relative separation of order $\alpha$ of $X$ by

$$
\overline{r e l_{\alpha}}(X):=\sup _{b \in \mathbb{R}^{d}} \#\left\{k \in \Lambda: x_{k} \in[-\alpha, \alpha]^{d}+b\right\},
$$

The following observation will be frequently used in the next sections. ${ }^{2}$

Observation 1.1.

(i) If $\alpha<\beta$, then

$$
r e l_{\alpha}(X) \leq \overline{r e l_{\alpha}}(X) \leq r e l_{\beta}(X) \leq\left\lceil\frac{\beta}{\alpha}\right\rceil^{d} \cdot \operatorname{rel}_{\alpha}(X) .
$$

Hence if $X$ is relatively separated, given $\alpha>0$ the amount of elements of $X$ in any cube of side $2 \alpha$ is bounded by a constant that depends only on $\alpha$.

(ii) If $X$ is uniformly separated, then it is relatively separated and

$$
\operatorname{rel}_{\alpha}(X) \leq\left\lceil\frac{2 \alpha}{\operatorname{sep}(X)}\right\rceil^{d} .
$$

(iii) If $X=\left\{x_{k}\right\}_{k \in \Lambda}$ is relatively separated and $Y=\left\{y_{k}\right\}_{k \in \Lambda}$ is uniformly close to $X$ (i.e. $\left.\delta:=\sup _{k \in \Lambda}\left|x_{k}-y_{k}\right|_{2}<\infty\right)$, then $Y$ is also relatively separated and

$$
\operatorname{rel}_{\alpha}(Y) \leq r e l_{\alpha+\delta}(X), \quad \overline{r e l_{\alpha}}(Y) \leq \overline{r e l_{\alpha+\delta}}(X) .
$$

(iv) Uniform and relative separation are translation invariant concepts; that is, for every set of points $X$ and $z \in \mathbb{R}^{d}$,

$$
\operatorname{sep}(X)=\operatorname{sep}(X+z) \text { and } \operatorname{rel}_{\alpha}(X)=\operatorname{rel}_{\alpha}(X+z) .
$$

(v) $X$ is relatively separated if and only if it is a finite union of uniformly separated sets. More precisely, $X \equiv\left\{x_{k}\right\}_{k \in \Lambda}$ is relatively separated if and only if there exists $\Lambda_{1}, \ldots, \Lambda_{n}$ such that $\Lambda=\bigcup_{j=1}^{n} \Lambda_{j}$ and for each $1 \leq j \leq$ $n, X_{j}:=\left\{x_{k}\right\}_{k \in \Lambda_{j}}$ is uniformly separated.

\footnotetext{
${ }^{2}$ For $t \in \mathbb{R}$ we denote by $\lceil t\rceil(\lfloor t\rfloor)$ the smallest (largest) integer that is bigger (smaller) than $t$.
} 
1.4. Amalgam spaces. In this section we introduce a family of function spaces that will be called amalgam spaces. This name is normally given to a much broader class of spaces of which the one we define is a particular example. We refer the reader to [9] for a thorough introduction to amalgam spaces in full generality and to [13] for an self-contained introduction to the spaces defined below.

Definition 1.4. For $1 \leq p, q \leq \infty$ and measurable $f: \mathbb{R}^{d} \rightarrow \mathbb{C}$ let us define,

$$
\|f\|_{W\left(L^{p}, L^{q}\right)}:=\left(\int_{\mathbb{R}^{d}}\left(\int_{[0,1]^{d}}|f(x+y)|^{p} d x\right)^{q / p} d y\right)^{1 / q},
$$

with the usual adjustments when $p$ or $q$ is $\infty$. Let us call amalgam space of parameters $p$ and $q$ the class of functions with finite $W\left(L^{p}, L^{q}\right)$ norm, identifying those functions that differ only in a set of measure zero.

$$
W\left(L^{p}, L^{q}\right):=\left\{f: \mathbb{R}^{d} \rightarrow \mathbb{C} \text { measurable }:\|f\|_{W\left(L^{p}, L^{q}\right)}<\infty\right\} .
$$

Observe that $\|f\|_{W\left(L^{p}, L^{q}\right)}:=\|\| f\left\|_{L^{p}\left([0,1]^{d}+x\right)}\right\|_{L^{q}(d x)}$. We say that $L^{p}$ is the local component of $W\left(L^{p}, L^{q}\right)$ while $L^{q}$ is its global component.

Endowed with the corresponding norms, each of these spaces is an isometrically translation invariant Banach space and we have the following inclusions:

If $1 \leq p_{1} \leq p_{0} \leq \infty$ and $1 \leq q_{0} \leq q_{1} \leq \infty$, then $W\left(L^{p_{0}}, L^{q_{0}}\right) \subseteq W\left(L^{p_{1}}, L^{q_{1}}\right)$.

In $W\left(L^{p}, L^{q}\right)$ there is an equivalent discrete norm (see [9]),

$$
\|f\|_{W\left(L^{p}, \ell q\right)}:=\left(\left.\left.\sum_{k \in \mathbb{Z}^{d}}\left|\int_{[0,1]^{d}}\right| f(x+k)\right|^{p} d x\right|^{\frac{q}{p}}\right)^{\frac{1}{q}},
$$

with the usual adjustments when $p$ or $q$ is $\infty$. Note that $\|f\|_{W\left(L^{p}, \ell^{q}\right)}$ is the $\ell^{q}$ norm of the sequence $\left(\|f\|_{L^{p}\left([0,1]^{d}+j\right)}\right)_{j \in \mathbb{Z}^{d}}$. This norm has some technical advantages over the continuous one, but with the drawback that translations are no longer isometries with respect to this norm.

When considered with the discrete norms we will write $W\left(L^{p}, \ell^{q}\right)$ instead of $W\left(L^{p}, L^{q}\right)$.

For $p<\infty$, we distinguish in $W\left(L^{\infty}, L^{p}\right)$ the (closed) subspace formed by all the continuous functions,

$$
W\left(C_{0}, L^{p}\right):=\left\{f \in W\left(L^{\infty}, L^{p}\right): \mathrm{f} \text { is continuous }\right\},
$$

and the corresponding discrete norm $\|\cdot\|_{W\left(C_{0}, \ell^{p}\right)}$ is just the restriction of $\|\cdot\|_{W\left(L^{\infty}, \ell^{p}\right)}$ to $W\left(C_{0}, L^{p}\right)$.

Similar notions are used for vector-valued functions: for $F: \mathbb{R}^{d} \rightarrow \mathbb{C}^{d}$, we write:

$$
\|F\|_{W\left(L^{p}, L^{q}\right)}=\left\||F(\cdot)|_{2}\right\|_{W\left(L^{p}, L^{q}\right)}, \quad\|F\|_{W\left(L^{p}, \ell^{q}\right)}=\left\||F(\cdot)|_{2}\right\|_{W\left(L^{p}, \ell^{q}\right)} .
$$

1.5. Irregular spline-type spaces. Given a function $\varphi \in W\left(C_{0}, \ell^{1}\right)$, a relatively separated subset of $\mathbb{R}^{d}, X \equiv\left\{x_{k}\right\}_{k \in \Lambda}$ and $1 \leq p<\infty$, we consider the closed linear space generated by the corresponding translates

$$
\mathcal{S}^{p}=\mathcal{S}^{p}(\varphi, X)=\overline{\operatorname{span}\left(\left\{\varphi\left(\cdot-x_{k}\right)\right\}_{k \in \Lambda}\right)},
$$

where the closure is taken in $L^{p}$ norm. 
When the set of translates of $\varphi,\left\{\varphi\left(\cdot-x_{k}\right)\right\}_{k \in \Lambda}$ is a p-Riesz sequence, we call $\mathcal{S}^{p}$ a principal spline-type space. One can also consider spline-type spaces generated by various functions. For simplicity we restrict ourselves to the case of just one generator. The results on this article can be easily generalized to finitely generated spaces.

When the set of points is a lattice, the space $\mathcal{S}^{p}$ has the additional structure of being invariant under a group of translations. If in addition $p=2, \mathcal{S}^{p}$ can be treated by fibration techniques (see [6], [7], [16].)

1.6. The sampling problem. We study the sampling problem in spline-type spaces. As shown below, the assumption that the generator $\varphi$ lies in $W\left(C_{0}, \ell^{1}\right)$ guarantees that the functions in $\mathcal{S}^{p}$ are continuous and therefore pointwise evaluation makes sense. Although this is true under weaker hypothesis, the requirement that the generator belongs to $W\left(C_{0}, \ell^{1}\right)$ has some important consequences such as a general oversampling phenomenon and the existence of effective reconstruction algorithms (see [2]). It is now a standard assumption in the literature.

Theorem 1.1. Let $\mathcal{S}^{p}$ be a spline-type space and $1 \leq p<\infty$. Then, there exists a constant $C$ such that for every $f \in \mathcal{S}^{p}$,

$$
\|f\|_{L^{p}} \leq\|f\|_{W\left(C_{0}, \ell^{p}\right)} \leq C\|f\|_{L^{p}} .
$$

In particular, $\mathcal{S}^{p} \subseteq W\left(C_{0}, \ell^{p}\right)$, and therefore every $f \in \mathcal{S}^{p}$ is continuous.

Remark 1.1. This theorem is usually stated in the case where the translates are regular (see for example [4]). Since we need this slightly more general version, we provide a proof. We will see that the constant $C>0$ depends only on $\varphi$ and the "regularity" of the set of nodes.

In order to prove the theorem, we will require the following lemma.

Lemma 1.2. If $f \in W\left(C_{0}, \ell^{1}\right)$ and $Y \equiv\left\{y_{k}\right\}_{k \in \Lambda}$ is a relatively separated set of points, then, using the symbol $I:=[0,1]^{d}$ one has:

$$
\sum_{k \in \Lambda} \sup _{I+y_{k}}|f| \leq 2^{d} \overline{r e l_{\frac{1}{2}}}(Y)\|f\|_{W\left(C_{0}, \ell^{1}\right)} .
$$

Proof.

$$
\begin{aligned}
\sum_{k \in \Lambda} \sup _{I+y_{k}}|f| & \leq \sum_{j \in \mathbb{Z}^{d}} \sum_{k: y_{k} \in I+j} \sup _{I+y_{k}}|f| \leq \sum_{j \in \mathbb{Z}^{d}} \overline{\operatorname{rel}_{\frac{1}{2}}}(Y) \sup _{I+I+j}|f| \\
& \leq \overline{\operatorname{rel}_{\frac{1}{2}}}(Y) \sum_{j \in \mathbb{Z}^{d}} \sum_{h \in\{0,1\}^{d}} \sup _{I+h+j}|f| \\
& \leq \overline{\operatorname{rel}_{\frac{1}{2}}}(Y) \sum_{h \in\{0,1\}^{d}} \sum_{j \in \mathbb{Z}^{d}} \sup _{I+h+j}|f|=\overline{\operatorname{rel}_{\frac{1}{2}}}(Y) 2^{d}\|f\|_{W\left(C_{0}, \ell^{1}\right)} .
\end{aligned}
$$

Now we are ready to prove Theorem 1.1.

Proof. Given $f \in S$, since $\left\{\varphi\left(\cdot-x_{k}\right)\right\}_{k}$ is a p-Riesz basis of $\mathcal{S}^{p}$, we write,

$$
f=\sum_{k \in \Lambda} c_{k} \varphi\left(\cdot-x_{k}\right)
$$

where $c \equiv\left(c_{k}\right)_{k \in \Lambda} \in \ell^{p}$ and the series (2) converges unconditionally in $L^{p}$. 
Given $j \in \mathbb{Z}^{d}$ and $x \in I+j$, observe that for each finite subset $\Lambda_{0} \subseteq \Lambda$,

$$
\left|\sum_{k \in \Lambda_{0}} c_{k} \varphi\left(x-x_{k}\right)\right| \leq \sum_{k \in \Lambda_{0}}\left|c_{k}\right| \sup _{I+j-x_{k}}|\varphi| \leq \sum_{k \in \Lambda}\left|c_{k}\right| \sup _{I+j-x_{k}}|\varphi| .
$$

Since the series (2) converges in $L^{p}(I+j)$, it has a subsequence that converges almost everywhere. Therefore for almost every $x \in I+j$ we have that $\left|\sum_{k \in \Lambda} c_{k} \varphi\left(x-x_{k}\right)\right| \leq$ $\sum_{k \in \Lambda}\left|c_{j}\right| \sup _{I+j-x_{k}}|\varphi|$.

Using the previous lemma and writing $Y_{j}:=\left\{j-x_{k}\right\}_{k \in \Lambda}$ for $j \in \mathbb{Z}^{d}$ we obtain:

$$
\begin{aligned}
\sum_{k \in \Lambda} \sup _{I+j-x_{k}}|\varphi| & \leq 2^{d} \overline{\operatorname{rel}_{\frac{1}{2}}}\left(Y_{j}\right)\|\varphi\|_{W\left(C_{0}, \ell^{1}\right)} \\
& =2^{d} \overline{\operatorname{rel}_{\frac{1}{2}}}(X)\|\varphi\|_{W\left(C_{0}, \ell^{1}\right)} .
\end{aligned}
$$

and for each $k \in \Lambda$ using the lemma with $Y_{k}^{\prime}:=\left\{j-x_{k}\right\}_{j \in \mathbb{Z}^{d}}$,

$$
\begin{aligned}
\sum_{j \in \mathbb{Z}^{d}} \sup _{I+j-x_{k}}|\varphi| & \leq 2^{d} \overline{\operatorname{rel}_{\frac{1}{2}}}\left(Y_{k}^{\prime}\right)\|\varphi\|_{W\left(C_{0}, \ell^{1}\right)} \\
& =2^{d} \overline{\operatorname{rel}_{\frac{1}{2}}}\left(\mathbb{Z}^{d}\right)\|\varphi\|_{W\left(C_{0}, \ell^{1}\right)} . \\
& =2^{2 d}\|\varphi\|_{W\left(C_{0}, \ell^{1}\right)} .
\end{aligned}
$$

Using this and Hölder's inequality (distinguishing the case $p=1$ ),

$$
\begin{aligned}
& \|f\|_{W\left(L^{p}, \ell^{\infty}\right)}^{p}=\sum_{j \in \mathbb{Z}^{d}} \sup _{I+j} \operatorname{ess}|f|^{p} \\
& \leq \sum_{j \in \mathbb{Z}^{d}}\left(\sum_{k \in \Lambda}\left|c_{k}\right| \sup _{I+j-x_{k}}|\varphi|\right)^{p} \\
& =\sum_{j \in \mathbb{Z}^{d}}\left(\sum_{k \in \Lambda}\left|c_{k}\right| \sup _{I+j-x_{k}}|\varphi|^{\frac{1}{p}} \sup _{I+j-x_{k}}|\varphi|^{\frac{1}{p^{\prime}}}\right)^{p} \\
& \leq \sum_{j \in \mathbb{Z}^{d}}\left(\sum_{k \in \Lambda}\left|c_{k}\right|^{p} \sup _{I+j-x_{k}}|\varphi|\right)\left(\sum_{k \in \Lambda} \sup _{I+j-x_{k}}|\varphi|\right)^{\frac{p}{p^{\prime}}} \\
& \leq\left(2^{d}\|\varphi\|_{W\left(C_{0}, \ell^{1}\right)} \overline{\operatorname{rel}_{\frac{1}{2}}}(X)\right)^{\frac{p}{p^{\prime}}} \sum_{j \in \mathbb{Z}^{d}} \sum_{k \in \Lambda}\left|c_{k}\right|^{p} \sup _{I+j-x_{k}}|\varphi| \\
& =\left(2^{d}\|\varphi\|_{W\left(C_{0}, \ell^{1}\right)} \overline{\operatorname{rel}_{\frac{1}{2}}}(X)\right)^{\frac{p}{p^{\prime}}} \sum_{k \in \Lambda}\left|c_{k}\right|^{p} \sum_{j \in \mathbb{Z}^{d}} \sup _{I+j-x_{k}}|\varphi| \\
& \leq \\
& \leq\left(2^{d}\|\varphi\|_{W\left(C_{0}, \ell^{1}\right)} \overline{\operatorname{rel}_{\frac{1}{2}}}(X)\right)^{\frac{p}{p^{\prime}}} 2^{2 d}\|\varphi\|_{W\left(C_{0}, \ell^{1}\right)}\|c\|_{p}^{p} .
\end{aligned}
$$

Therefore,

$$
\|f\|_{L^{p}} \leq\|f\|_{W\left(L^{\infty}, \ell^{p}\right)} \leq 2^{\frac{d}{p}+d} \overline{\operatorname{rel}_{\frac{1}{2}}}(X)^{\frac{1}{p^{p}}}\|\varphi\|_{W\left(C_{0}, \ell^{1}\right)}\|c\|_{p} \leq C\|f\|_{L^{p}},
$$

where the last inequality follows from the equivalence between the $L^{p}$ norm of a function in $\mathcal{S}^{p}$ and its coefficients. Hence $f \in W\left(L^{\infty}, \ell^{p}\right)$ and the $W\left(L^{\infty}, \ell^{p}\right)$ and $L^{p}$ norms are equivalent on $\mathcal{S}^{p}$. Since $\varphi$ is continuous so are the final partial sums. Since $W\left(L^{\infty}, \ell^{p}\right)$ implies uniform convergence it follows that $f$ is continuous. 
Remark 1.2. Using the general theory developed in [9], Theorem 1.1 follows easily. Since $\left\{x_{k}\right\}_{k \in \Lambda}$ is relatively separated, if $c \in \ell^{p}$, the measure $\sum_{k} c_{k} \delta_{x_{k}}$ belongs to $W\left(M, \ell^{p}\right)$ (and conversely). Here $M$ is the space of complex-valued bounded measures under the variation norm. The theorem now follows from the convolution relation (together with corresponding norm estimates):

$$
W\left(M, L^{p}\right) * W\left(C_{0}, L^{1}\right) \subseteq W\left(C_{0}, L^{p}\right) .
$$

We have just shown that in a spline-type space functions can be evaluated. The next lemma shows that evaluation on a relatively separated set of points is continuous.

Lemma 1.3. Let $\mathcal{S}^{p}$ be a spline-type space and $1 \leq p<\infty$. Let $Y \equiv\left\{y_{k}\right\}_{k \in \Lambda} \subseteq \mathbb{R}^{d}$ be a relatively separated set of points. Then, the sampling operator

$$
\begin{aligned}
\mathcal{S}^{p} & \rightarrow \ell^{p}(\Lambda) \\
f & \mapsto\left(f\left(y_{k}\right)\right)_{k \in \Lambda}
\end{aligned}
$$

is well-defined and bounded, with a bound that does not depend on $p$.

Proof. Let $I:=[0,1]^{d}$.

$$
\begin{aligned}
\|\left(f\left(y_{k}\right)_{k \in \Lambda} \|_{p}^{p}\right. & =\sum_{k \in \Lambda}\left|f\left(y_{k}\right)\right|^{p} \leq \sum_{j \in \mathbb{Z}^{d}} \sum_{y_{k} \in I+j}\left|f\left(y_{k}\right)\right|^{p} \\
& \leq \sum_{j \in \mathbb{Z}^{d}} \overline{\operatorname{rel}_{\frac{1}{2}}}(Y)\left(\sup _{I+j}|f|\right)^{p}=\overline{\operatorname{rel}_{\frac{1}{2}}}(Y)\|f\|_{W\left(C_{0}, \ell^{p}\right)}^{p} .
\end{aligned}
$$

Then, by Theorem 1.1 we have,

$$
\|\left(f\left(y_{k}\right)_{k \in \Lambda}\left\|_{p} \leq \overline{\operatorname{rel}_{\frac{1}{2}}}(Y)^{\frac{1}{p}}\right\| f\left\|_{W\left(C_{0}, \ell^{p}\right)} \leq C\right\| f \|_{L^{p}} .\right.
$$

Definition 1.5. A set $Y \equiv\left\{y_{k}\right\}_{k \in \Lambda}$ in $\mathbb{R}^{d}$ is called a sampling set for $\mathcal{S}^{p}$ if there exists $A, B>0$ such that for every $f \in \mathcal{S}^{p}$,

$$
A\|f\|_{L^{p}} \leq\left\|\left(f\left(y_{k}\right)\right)_{k \in \Lambda}\right\|_{\ell^{p}} \leq B\|f\|_{L^{p}} .
$$

If $Y$ is relatively separated, we define its sampling operator $\Upsilon_{Y}: \ell^{p}(\Lambda) \rightarrow \ell^{p}(\Lambda)$ by

$$
\Upsilon_{Y}(c)=\left(z_{j}\right)_{j \in \Lambda}, \quad z_{j}:=\sum_{k \in \Lambda} c_{k} \varphi\left(y_{j}-x_{k}\right) .
$$

Observation 1.2. According to the definition, $Y$ is a sampling set if and only if its sampling operator, $\Upsilon_{Y}$ is bounded below.

In addition, if $p=2$, since each evaluation map $f \mapsto f(x)$ is a continuous linear functional on $\mathcal{S}^{p}$, by Riesz's Theorem it is represented by its reproducing kernel $K_{x} \in \mathcal{S}^{p}$, called the reproducing kernel in x. Therefore, by equation (3) we observe that $Y \equiv\left\{y_{k}\right\}_{k \in \Lambda}$ is a sampling set if and only if the family of reproducing kernels $\left\{K_{y_{k}}\right\}_{k \in \Lambda}$ is a frame of $\mathcal{S}^{p}$. 


\section{Perturbation of SAmpling Sets}

In this section we will work in a spline-type space $\mathcal{S}^{p}$, so we will make the following assumption.

\section{General assumption for section 2}

- $\varphi \in W\left(L^{1}, C_{0}\right)$

- $1 \leq p<\infty$ and $X \equiv\left\{x_{k}\right\}_{k \in \Lambda} \subseteq \mathbb{R}^{d}$ is a relatively separated set of points, where the set of indexes $\Lambda$ is countable

- The set $\left\{\varphi\left(\cdot-x_{k}\right)\right\}_{k \in \Lambda}$ is a p-Riesz basis of its closed linear span, $\mathcal{S}^{p}$.

When the set of points $X$ is a lattice, this last hypothesis is known to be equivalent to a certain condition on the periodization of $\hat{\varphi}$ (see [4]).

We will consider sampling sets $Y \equiv\left\{y_{k}\right\}_{k \in \Lambda} \subseteq \mathbb{R}^{d}$ and study the possibility of slightly disturbing them while still retaining its sampling-set properties.

Definition 2.1. Let $Y=\left\{y_{k}\right\}_{k \in \Lambda}$ be a relatively separated sampling set for $\mathcal{S}^{p}$. We define its perturbation radius $\rho(Y)$ as the supremum of all $L \geq 0$ such that every set $\left\{z_{k}\right\}_{k \in \Lambda}$ such that $\sup _{k \in \Lambda}\left|z_{k}-y_{k}\right|_{2} \leq L$, is a sampling set for $\mathcal{S}^{p}$.

Note that the supremum is taken over an interval that contains 0 (it may be just $\{0\})$. Clearly, if $Z \equiv\left\{z_{k}\right\}_{k \in \Lambda}$ verifies $\sup _{k \in \Lambda}\left|z_{k}-y_{k}\right|_{2}<\rho(Y)$, then $Z$ is also a sampling set for $\mathcal{S}^{p}$.

2.1. General results. In this section we show that the perturbation radius of a sampling set is always positive under the only hypothesis of continuity and decay of the generator. The central technique will be to imitate Young's convolution inequality, replacing every equality that depends on the group structure of the set of points by an estimation based on the decay of the generator. In order to do this, we will show that the generator has a certain uniform continuity property that will allow us to estimate its variation in terms of the separation and distance between the sets of points. The importance of these estimations is that they are invariant under arbitrary translations.

Theorem 2.1. Suppose that $Y \equiv\left\{y_{k}\right\}_{k \in \Lambda} \subseteq \mathbb{R}^{d}$ is a relatively separated sampling set for $\mathcal{S}^{p}$. Then $Y$ has strictly positive perturbation radius $\rho(Y)>0$. Hence, if $Z \equiv\left\{z_{k}\right\}_{k \in \Lambda}$ is such that $\sup _{k \in \Lambda}\left|z_{k}-y_{k}\right|_{2}<\rho(Y)$, then $Z$ is also a sampling set for $\mathcal{S}^{p}$.

To prove this, we will study the map that sends a (relatively separated) set of points to its sampling operator.

Definition 2.2. Let $\Delta$ be the set of all $\Lambda$-indexed sets of $\mathbb{R}^{d}$ that are relatively separated.

$$
\Delta:=\left\{Z \equiv\left(z_{k}\right)_{k \in \Lambda}:\left\{z_{k}\right\}_{k \in \Lambda} \subseteq \mathbb{R}^{d} \text { is a relatively separated set }\right\} .
$$

For $M>0$, let us define

$$
\Delta_{M}:=\left\{Z \in \Delta: \operatorname{rel}_{\frac{1}{2}}(Z) \leq M\right\}
$$

That is, $\Delta_{M}$ is the set of those $Z \in \Delta$ such that every cube of measure 1 contains at most $M$ elements of $Z$ (to be more precise, for every cube of measure 1 , there are at most $M$ indexes such that the corresponding elements of $Z$ belong to the cube.) 
For technical reasons we will also define,

$$
\Delta_{\bar{M}}:=\left\{Z \in \Delta: \overline{\operatorname{rel}_{\frac{1}{2}}}(Z) \leq M\right\} .
$$

Observe that $\Delta=\bigcup_{M>0} \Delta_{M}$ and that $\Delta_{\bar{M}} \subseteq \Delta_{M} \subseteq \Delta_{\overline{2^{-d} M}}$.

Let us consider the uniform distance on $\Delta$,

$$
d_{\infty}\left(Z, Z^{\prime}\right):=\sup _{k \in \Lambda}\left|z_{k}-z_{k}^{\prime}\right|_{2} \text {, where } Z, Z^{\prime} \in \Delta .
$$

To be rigorous, $d_{\infty}$ is not a distance on $\Delta$ since it can take the value $\infty$. To overcome this problem we will consider

$$
d_{\infty}^{*}\left(Z, Z^{\prime}\right):=\sup _{k \in \Lambda} \min \left\{\left|z_{k}-z_{k}^{\prime}\right|_{2}, 1\right\}, \text { where } Z, Z^{\prime} \in \Delta .
$$

This is a distance on $\Delta$ and for $\delta<1, d_{\infty}\left(Z, Z^{\prime}\right) \leq \delta$ if and only if $d_{\infty}^{*}\left(Z, Z^{\prime}\right) \leq \delta$. Hence, for local considerations we can use $d_{\infty}$.

Each $Z \in \Delta$ is a relatively separated set, so its sampling operator $\Upsilon_{Z}$ is well defined and bounded. We can therefore consider the map

$$
\Upsilon: \Delta \rightarrow B\left(\ell^{p}(\Lambda)\right),
$$

that sends a $(\Lambda$-indexed, relatively separated) set of points to its sampling operator, defined in (4). Here, $B\left(\ell^{p}(\Lambda)\right)$ denotes the set of bounded operators on $\ell^{p}(\Lambda)$. Although it is not emphasized in the notation, the map $\Upsilon$ depends on $X$ and $\varphi$. Theorem 2.1 will follow easily once we have proved that the map $\Upsilon$ is continuous.

Let us note that an arbitrary complex-valued function $f: \mathbb{R}^{d} \rightarrow \mathbb{C}$ induces pointwise a function on $\Delta$ that we will also denote $f$. That is,

$$
f: \Delta \rightarrow \mathbb{C}^{\Lambda}, f(Z):=\left(f\left(z_{k}\right)\right)_{k \in \Lambda} .
$$

For each $Z \in \Delta$, its sampling operator $\Upsilon_{Z}$ depends on the values that the generator $\varphi$ takes on the translations of $Z,\left\{Z+x_{k}\right\}_{k \in \Lambda}$. In order to study the behavior of $\Upsilon$ near $Z$ we must consider every translation (in $X$ ) of the sets of points that are uniformly close to $Z$. These sets share a similar relative separation and so do their arbitrary translations.

The importance of the amalgam space $W\left(C_{0}, \ell^{1}\right)$ in this study of perturbability is that its induced functions on $\Delta$ have an uniform modulus of continuity over families of sets with a bounded relative separation. See [12] for related results an a discussion of amalgam spaces as a tool for analyzing robustness in spline-type spaces.

Definition 2.3. Given $f \in W\left(C_{0}, \ell^{1}\right), \delta, M>0$ we define the $\delta, M$-discrete oscillation of $f$ by

$$
O_{\delta, M}(f):=\sup \left\{\|f(Y)-f(Z)\|_{1}: Y \in \Delta_{M}, d_{\infty}(Y, Z) \leq \delta\right\},
$$

and its strong version:

$$
O_{\delta, \bar{M}}(f):=\sup \left\{\|f(Y)-f(Z)\|_{1}: Y \in \Delta_{\bar{M}}, d_{\infty}(Y, Z) \leq \delta\right\} .
$$

Lemma 2.2. Let $f \in W\left(C_{0}, \ell^{1}\right)$, the following statements hold:

(a) For each $\delta, M>0: 0 \leq O_{\delta, \bar{M}}(f) \leq O_{\delta, M}(f) \leq O_{\delta, \overline{2^{-d} M}}(f)<\infty$.

(b) For each $M>0, O_{\delta, \bar{M}}(f) \longrightarrow 0$, as $\delta \rightarrow 0$. 
(c) The induced application

$$
f: \Delta \rightarrow l^{1}(\Lambda), f(Y):=\left(f\left(y_{k}\right)\right)_{k \in \Lambda}
$$

is well-defined and continuous. Moreover, for every $M>0, f$ is uniformly continuous on $\Delta_{\bar{M}}$.

Proof. Let $I:=[0,1]^{d}$ be the unitary cube and also set $I^{\prime}:=I+[-1,1]^{d}=[-1,2]^{d}$ and $I^{\prime \prime}:=I^{\prime}+[-1,1]^{d}=[-2,3]^{d}$.

We first observe that the induced function $f$ takes values in $l^{1}(\Lambda)$. If $Z \in \Delta$,

$$
\begin{aligned}
\|f(Y)\|_{1} & =\sum_{k \in \Lambda}\left|f\left(y_{k}\right)\right| \leq \sum_{j \in \mathbb{Z}^{d}} \sum_{y_{k} \in I+j}\left|f\left(y_{k}\right)\right| \\
& \leq \sum_{j \in \mathbb{Z}^{d}} \sup _{I+j}|f| \#\left\{k \in \Lambda: y_{k} \in I+j\right\} \\
& \leq \overline{\operatorname{rel}_{\frac{1}{2}}}(Y)\|f\|_{W\left(C_{0}, \ell^{1}\right)}<\infty .
\end{aligned}
$$

So, $f(Z) \in l^{1}(\Lambda)$.

Now we apply this last estimate to establish (a). For $Y \in \Delta_{\bar{M}}$ and $d_{\infty}(Y, Z) \leq \delta$,

$$
\|f(Y)-f(Z)\|_{1} \leq\|f(Y)\|_{1}+\|f(Z)\|_{1} \leq\left(\overline{\operatorname{rel}_{\frac{1}{2}}}(Y)+\overline{\operatorname{rel}_{\frac{1}{2}}}(Z)\right)\|f\|_{W\left(C_{0}, \ell^{1}\right)} .
$$

According to Observation 1.1,

So,

$$
\overline{\operatorname{rel}_{\frac{1}{2}}}(Z) \leq \overline{\operatorname{rel}_{\frac{1}{2}+\delta}}(Y) \leq\lceil 1+2 \delta\rceil^{d} \overline{\operatorname{rel}_{\frac{1}{2}}}(Y) .
$$

$$
\|f(Y)-f(Z)\|_{1} \leq\left(\lceil 1+2 \delta\rceil^{d}+1\right) \overline{\operatorname{rel}_{\frac{1}{2}}}(Y)\|f\|_{W\left(C_{0}, \ell^{1}\right)} .
$$

Hence, $O_{\delta, \bar{M}}(f) \leq\left(\lceil 1+2 \delta\rceil^{d}+1\right) M\|f\|_{W\left(C_{0}, \ell^{1}\right)}<\infty$.

Let us now prove (b). Let $M>0$ and $\varepsilon>0$ be given, we look for $\delta_{0}>0$ such that: $\left\|f(Z)-f\left(Z^{\prime}\right)\right\|_{1} \leq \varepsilon$, whenever $Z \in \Delta_{\bar{M}}$ and $d_{\infty}\left(Z, Z^{\prime}\right) \leq \delta_{0}$. This will show that $O_{\delta, \bar{M}}(f) \leq \epsilon$ if $0<\delta \leq \delta_{0}$.

Since $f \in W\left(C_{0}, \ell^{1}\right)$, there exists a cube $Q \subseteq \mathbb{R}^{d}$ of side $2 l, l>0$ such that:

$$
\sum_{k \in \mathbb{Z}^{d} \backslash Q} \sup _{I+k}|f|<\frac{\varepsilon}{2\left(3^{d}+1\right) M} .
$$

Since $f$ is continuous and $Q+I^{\prime \prime}$ is compact, there exists $\delta_{0}, 0<\delta_{0}<1$, such that

$$
\text { if } x, y \in Q+I^{\prime \prime} \text { and }|x-y| \leq \delta_{0} \text {, then }|f(x)-f(y)|<\frac{\varepsilon}{2(\lceil 2 l+3\rceil)^{d} M} \text {. }
$$

To see that $\delta_{0}$ is adequate, let $Z \in \Delta_{M}$ and $Z^{\prime} \in \Delta$ be such that $d_{\infty}\left(Z, Z^{\prime}\right) \leq \delta_{0}$.

Let $\Lambda_{0}:=\left\{k \in \Lambda: z_{k} \in Q+I^{\prime}\right\}$. Since the side of $Q+I^{\prime}$ measures $2 l+3$, according to Observation 1.1,

$$
\# \Lambda_{0} \leq \overline{\operatorname{rel}_{l+\frac{3}{2}}}(Z) \leq(\lceil 2 l+3\rceil)^{d} \overline{\operatorname{rel}_{\frac{1}{2}}}(Z) \leq(\lceil 2 l+3\rceil)^{d} M .
$$

Since $d_{\infty}\left(Z, Z^{\prime}\right) \leq \delta_{0}<1$,

$$
\begin{aligned}
& \text { if } k \in \Lambda_{0}, \quad z_{k}, z_{k}^{\prime} \in Q+I^{\prime \prime}, \\
& \text { if } k \notin \Lambda_{0}, \quad z_{k}, z_{k}^{\prime} \notin Q+I .
\end{aligned}
$$

It also follows by Observation 1.1 that

$$
\overline{\operatorname{rel}_{\frac{1}{2}}}\left(Z^{\prime}\right) \leq \overline{\operatorname{rel}_{3}}(Z) \leq 3^{d} M
$$


Now,

$$
\begin{aligned}
\sum_{k \in \Lambda} \mid & f\left(z_{k}\right)-f\left(z_{k}^{\prime}\right)\left|=\sum_{k \in \Lambda_{0}}\right| f\left(z_{k}\right)-f\left(z_{k}^{\prime}\right)\left|+\sum_{k \in \Lambda \backslash \Lambda_{0}}\right| f\left(z_{k}\right)-f\left(z_{k}^{\prime}\right) \mid \\
& \leq \# \Lambda_{0} \sup _{\substack{x, y \in Q+I^{\prime \prime} \\
|x-y| \leq \delta}}|f(x)-f(y)|+\sum_{z_{k} \notin Q+I}\left|f\left(z_{k}\right)\right|+\sum_{z_{k}^{\prime} \notin Q+I}\left|f\left(z_{k}^{\prime}\right)\right| \\
& \leq \frac{\varepsilon}{2}+\sum_{j \in \mathbb{Z}^{d}} \sum_{z_{k} \in I+j \backslash Q+I}\left|f\left(z_{k}\right)\right|+\sum_{j \in \mathbb{Z}^{d}} \sum_{z_{k}^{\prime} \in I+j \backslash Q+I}\left|f\left(z_{k}^{\prime}\right)\right| \\
& \leq \frac{\varepsilon}{2}+\sum_{j \in \mathbb{Z}^{d} \backslash Q} \sum_{z_{k} \in I+j}\left|f\left(z_{k}\right)\right|+\sum_{j \in \mathbb{Z}^{d} \backslash Q} \sum_{z_{k}^{\prime} \in I+j}\left|f\left(z_{k}^{\prime}\right)\right| \\
& \leq \frac{\varepsilon}{2}+\sum_{j \in \mathbb{Z}^{d} \backslash Q} \overline{\operatorname{rel}_{\frac{1}{2}}}(Z) \sup _{I+j}|f|+\sum_{j \in \mathbb{Z}^{d} \backslash Q} \frac{\operatorname{rel}_{\frac{1}{2}}}{Z^{\prime}}\left(Z^{\prime}\right) \sup _{I+j}|f| \\
& \leq \frac{\varepsilon}{2}+\left(3^{d}+1\right) M \sum_{j \in \mathbb{Z}^{d} \backslash Q} \sup _{I+j}|f| \\
& <\frac{\varepsilon}{2}+\frac{\varepsilon}{2}=\varepsilon .
\end{aligned}
$$

The fact that $f$ is uniformly continuous on each $\Delta_{M}$ follows immediately from (b) so it only remains to show that $f$ is continuous all over $\Delta$. It suffices to show that $f$ is continuous on every ball $B=B(Z, r)$ with $Z \in \Delta$ and $r>0$, but for each $Z^{\prime} \in B, \overline{\operatorname{rel}_{\frac{1}{2}}}\left(Z^{\prime}\right) \leq \overline{\operatorname{rel}_{\frac{1}{2}+r}}(Z)=: M$, and so $B \subseteq \Delta_{M}$ where we already know that $f$ is (uniformly) continuous.

Now we can prove the main estimate of the section, that together with Lemma 2.2 will prove Theorem 2.1 .

Theorem 2.3. Let $\delta>0$ and let $Y \in \Delta_{M}$ and $Z \in \Delta$ be such that $d_{\infty}(Y, Z) \leq \delta$. Let $\Upsilon_{Y}, \Upsilon_{Z}$ be their respective sampling operators. Let $N:=\operatorname{rel}_{\frac{1}{2}}(X)$. Then, the following estimate holds:

$$
\left\|\Upsilon_{Y}-\Upsilon_{Z}\right\|_{p} \leq O_{\delta, M}(\varphi)^{\frac{1}{p}} O_{\delta, N}(\varphi)^{\frac{1}{p^{\prime}}}
$$


Proof. We consider first the case $p>1$. For $c \in \ell^{p}(\Lambda)$,

$$
\begin{aligned}
& \|\left.\left(\Upsilon_{Y}-\Upsilon_{Z}\right)(c)\right|^{p}=\sum_{j \in \Lambda}\left|\sum_{k \in \Lambda} c_{k}\left[\varphi\left(y_{j}-x_{k}\right)-\varphi\left(z_{j}-x_{k}\right)\right]\right|^{p} \\
& \quad \leq \sum_{j \in \Lambda}\left(\sum_{k \in \Lambda}\left|c_{k}\right|\left|\varphi\left(y_{j}-x_{k}\right)-\varphi\left(z_{j}-x_{k}\right)\right|^{1 / p}\left|\varphi\left(y_{j}-x_{k}\right)-\varphi\left(z_{j}-x_{k}\right)\right|^{1 / p^{\prime}}\right)^{p} \\
& \quad \leq \sum_{j \in \Lambda}\left(\sum_{k \in \Lambda}\left|c_{k}\right|^{p}\left|\varphi\left(y_{j}-x_{k}\right)-\varphi\left(z_{j}-x_{k}\right)\right|\right)\left(\sum_{k \in \Lambda}\left|\varphi\left(y_{j}-x_{k}\right)-\varphi\left(z_{j}-x_{k}\right)\right|\right)^{p / p^{\prime}} \\
& \quad \leq \sum_{j \in \Lambda}\left(\sum_{k \in \Lambda}\left|c_{k}\right|^{p}\left|\varphi\left(y_{j}-x_{k}\right)-\varphi\left(z_{j}-x_{k}\right)\right|\right) O_{\delta, N}(\varphi)^{p / p^{\prime}} \\
& \quad \text { for each } j, \operatorname{rel}_{\frac{1}{2}}\left(\left(y_{j}-x_{k}\right)_{k}\right)=\operatorname{rel}_{\frac{1}{2}}(X)=N \text { and } d_{\infty}\left(\left(y_{j}-x_{k}\right)_{k},\left(z_{j}-x_{k}\right)_{k}\right)=\left|y_{j}-z_{j}\right| \leq \delta \\
& \quad \leq O_{\delta, N}(\varphi)^{p / p^{\prime}} \sum_{k \in \Lambda}\left|c_{k}\right|^{p} \sum_{j \in \Lambda}\left|\varphi\left(y_{j}-x_{k}\right)-\varphi\left(z_{j}-x_{k}\right)\right| \\
& \quad \leq O_{\delta, N}(\varphi)^{p / p^{\prime}} O_{\delta, M}(\varphi) \sum_{k \in \Lambda}\left|c_{k}\right|^{p} \\
& \quad \text { for each } k, \operatorname{rel} \frac{1}{2}\left(\left(y_{j}-x_{k}\right)_{j}\right)=\operatorname{rel}_{\frac{1}{2}}(Y) \leq M \text { and } d_{\infty}\left(\left(y_{j}-x_{k}\right)_{j},\left(z_{j}-x_{k}\right)_{j}\right)=d_{\infty}(Y, Z) \leq \delta \\
& \quad=O_{\delta, N}(\varphi)^{p / p^{\prime}} O_{\delta, M}(\varphi)\|c\|_{p}^{p} .
\end{aligned}
$$

Hence, $\left\|\Upsilon_{Y}-\Upsilon_{Z}\right\| \leq O_{\delta, N}(\varphi)^{\frac{1}{p^{\prime}}} O_{\delta, M}(\varphi)^{\frac{1}{p}}$.

A similar calculation proves that the same estimate holds in the case $p=1$ (interpreting $p / p^{\prime}=0$ as usual in this case).

We have one of the main results of this section.

Theorem 2.4. The map

$$
\Upsilon:\left(\Delta, d_{\infty}^{*}\right) \rightarrow\left(B\left(\ell^{p}(\Lambda)\right),\|\cdot\|\right),
$$

is continuous. Here the norm in $B\left(\ell^{p}(\Lambda)\right.$ is the operator norm.

Proof. Given $Y \in \Delta$ let us see that $\Upsilon$ is continuous in $Y$. Let $M:=\overline{\operatorname{rel}_{\frac{1}{2}}}(Y)$ and $N:=\overline{\operatorname{rel}_{\frac{1}{2}}}(X)$. For $Z \in \Delta$, set $\delta:=d_{\infty}(Y, Z)$ and observe that $d_{\infty}^{*}(Y, Z) \rightarrow 0$ if and only if $\delta \rightarrow 0$. Combining Lemma 2.2 with Theorem 2.3 we have that,

$$
\left\|\Upsilon_{Y}-\Upsilon_{Z}\right\| \leq O_{\delta, N}(\varphi)^{\frac{1}{p^{\prime}}} O_{\delta, M}(\varphi)^{\frac{1}{p}} \longrightarrow 0, \text { as } d_{\infty}^{*}(Y, Z) \rightarrow 0 .
$$

Now Theorem 2.1 follows easily. We restate it here for convenience of the reader.

Theorem (2.1). Suppose that $Y \equiv\left\{y_{k}\right\}_{k \in \Lambda} \subseteq \mathbb{R}^{d}$ is a relatively separated sampling set for $\mathcal{S}^{p}$. Then, $\rho(Y)>0$. Hence, if $Z \equiv\left\{z_{k}\right\}_{k \in \Lambda}$ is such that $\sup _{k \in \Lambda}\left|z_{k}-y_{k}\right|_{2}<$ $\rho(Y)$, then $Z$ is also a sampling set for $\mathcal{S}^{p}$. 
Proof. Let us first observe that Theorem 2.1 simply states that the subset of $\left(\Delta, d_{\infty}\right)$ formed by the sampling sets is open. Indeed, $\rho$ is the radius of a ball centered at $Y$ that contains only sampling sets. (Remember that, according to Observation 1.1, every set at a finite $d_{\infty}$ distance of $Y$ will necessarily be relatively separated.)

Consider $B B$, the subset of $B\left(\ell^{p}\right)$ formed by the operators that are boundedbelow. $B B$ is an open subset of $B\left(\ell^{p}\right)$ since, if $T$ is a bounded-below operator with lower bound $A>0$, every operator $U$ such that $\|T-U\|<A$ is also bounded below.

Finally note that the set of all sampling sets is exactly the inverse image of $B B$ through $\Upsilon$, and so it is an open set.

2.2. Some estimates. The result in the previous subsection shows that a sampling set can be disturbed by a small amount $\rho$ without losing its sampling properties. We now study, adding some hypothesis on the generator function, the behavior of $\rho$ when the parameters of the problem vary. Similar estimates for bandlimited functions have been derived in [10] (see also [11]).

We will be able to estimate the modulus of uniform continuity in the sense of Lemma 2.2 of the generator and quantize the dependence of $\rho$ on the lower bound of the sampling operator and the separation of the sets of points involved.

Although the results yield good estimates in some examples, they tend to be less accurate with smoother generator functions. It remains to extend the results in this section to allow for better estimates in the case of smooth generators.

We would like to point out that the estimates in this sections unfortunately do not account for the fact that adding a point to a sampling set increases (or at least does not decrease) its perturbation radius.

Theorem 2.5. Suppose that $\varphi$ has a weak derivative, such that $|\nabla(\varphi)|_{2} \in W\left(L^{\infty}, \ell^{1}\right)$. Let $Y \in \Delta_{M}$ be a sampling set. Set

$$
A:=\inf _{\|c\|=1}\left\|\Upsilon_{Y}(c)\right\|, N:=\operatorname{rel}_{\frac{1}{2}}(X) .
$$

and

$$
L:=\sup \left\{\delta>0: \delta\lceil 1+2 \delta\rceil^{d} N^{1 / p^{\prime}} M^{1 / p}\|\nabla(\varphi)\|_{W\left(L^{\infty}, \ell^{1}\right)} \leq A\right\} .
$$

Then $\rho(Y) \geq L$, (where $\frac{1}{p^{\prime}}=0$ if $p=1$ ).

In particular, for a uniformly separated set $Y$, if we have upper bounds for sep $(Y)$ and $\rho(Y)$,

$$
\rho(Y) \geq L \approx A \operatorname{sep}(Y)^{d / p}
$$

Note that by Proposition $1.2, A \neq 0$.

In specific examples, there are (good) upper bounds for the separation of sampling sets. For example, if the generator has compact support, the maximum gap between points of any sampling set can be easily estimated in terms of the support of the generator (see for example, [3]). This also sets an upper bound on the perturbation radius, since a big perturbation radius would imply the existence of sampling sets with a big maximum gap.

Under weaker conditions we get a similar estimation.

Theorem 2.6. Suppose that $\varphi$ has a weak derivative, such that $|\nabla(\varphi)|_{2} \in W\left(L^{q}, \ell^{1}\right)$, $d<q<\infty$. Then, there exists a constant $C$ that depends only on $d$ and $q$ such 
that, for every relatively separated sampling set $Y \equiv\left\{y_{k}\right\}_{k \in \Lambda} \subseteq \mathbb{R}^{d}$ we have

$$
\rho(Y) \geq L,
$$

where

$$
A:=\inf _{\|c\|=1}\left\|\Upsilon_{Y}(c)\right\|, N:=\operatorname{rel}_{\frac{1}{2}}(X)
$$

and

$$
L:=\sup \left\{\delta>0: C \delta^{1-\frac{d}{q}}(1+2\lceil\delta / 2\rceil)^{2 d} M^{\frac{1}{p}} N^{\frac{1}{p^{\prime}}}\|\nabla(f)\|_{W\left(L^{q}, \ell^{1}\right)} \leq A\right\} .
$$

In particular, for a uniformly separated set $Y$, if we have upper bounds for sep $(Y)$ and $\rho(Y)$,

$$
\rho(Y) \geq L \approx\left(A \operatorname{sep}(Y)^{d / p}\right)^{1+\frac{d}{q-d}} .
$$

Remark 2.1. The constant $C$ can be explicitly found.

Both theorems will follow once we have estimated the discrete oscillation of the generator function. We do so in the following lemmas.

We will consider the Sobolev space $W_{1}^{p}\left(\mathbb{R}^{d}\right)$ of all functions in $L^{p}$ with a weak derivative in $L^{p}$. As shown by the proof of the following lemma, a function $f$ with a bounded weak derivative can be changed in a null-measure set so that it becomes continuous. The continuity hypothesis in the next lemma just asserts that we have chosen that continuous representative.

Lemma 2.7. Let $f \in W_{1}^{p}\left(\mathbb{R}^{d}\right)$ be continuous and such that $|\nabla(f)|_{2} \in L^{\infty}\left(\mathbb{R}^{d}\right)$. Let $x, y \in \mathbb{R}^{d}$ and let $B$ be the ball of diameter $|x-y|_{2}$ centered in the middle point between $x$ and $y$. Then,

$$
|f(x)-f(y)| \leq \sup _{B} \operatorname{ess}\|\nabla(f)\|_{2}|x-y|_{2} .
$$

Proof. Let $\eta$ be a smooth, positive kernel, that is supported in $B_{1}(0)$ and has integral 1. Let $\eta_{\varepsilon}=\frac{1}{\varepsilon^{d}} \eta(\dot{\bar{\varepsilon}})$ and $f_{\varepsilon}=f * \eta_{\varepsilon}$.

Let $x^{\prime}, y^{\prime} \in B^{\circ}$ be arbitrary points. Let $B^{\prime} \subset B^{\circ}$ be a closed ball that contains $x^{\prime}$ and $y^{\prime}$. By the mean value theorem, there exists $c \in B^{\prime}$ such that,

$$
\left|f_{\varepsilon}\left(x^{\prime}\right)-f_{\varepsilon}\left(y^{\prime}\right)\right| \leq\left|\nabla\left(f_{\varepsilon}\right)(c)\right|_{2}\left|x^{\prime}-y^{\prime}\right|_{2} .
$$

If $0<\varepsilon<d\left(B^{\prime}, B\right)$, then $B_{\epsilon}(c) \subseteq B$ and we can estimate,

$$
\begin{aligned}
\left|\nabla\left(f_{\varepsilon}\right)(c)\right|_{2} & =\left|\nabla(f) * \eta_{\varepsilon}(c)\right|_{2} \leq\left(|\nabla(f)|_{2} * \eta_{\varepsilon}\right)(c) \\
& \leq \sup _{B_{\varepsilon}(c)} \operatorname{ess}|\nabla(f)|_{2}\left\|\eta_{\varepsilon}\right\|_{1} \leq \sup _{B} \operatorname{ess}|\nabla(f)|_{2} .
\end{aligned}
$$

So,

$$
\left|f_{\varepsilon}\left(x^{\prime}\right)-f_{\varepsilon}\left(y^{\prime}\right)\right| \leq \sup _{B} \operatorname{ess}|\nabla(f)|_{2}\left|x^{\prime}-y^{\prime}\right|_{2} .
$$

Since $f$ is continuous, $x^{\prime}$ and $y^{\prime}$ are Lebesgue points of $f$. Letting $\varepsilon \rightarrow 0^{+}$we obtain that

$$
\left|f\left(x^{\prime}\right)-f\left(y^{\prime}\right)\right| \leq \sup _{B} \operatorname{ess}|\nabla(f)|_{2}\left|x^{\prime}-y^{\prime}\right|_{2} .
$$

The lemma follows by letting $x^{\prime} \rightarrow x$ and $y^{\prime} \rightarrow y$. 
Lemma 2.8. Let $f \in W\left(C_{0}, \ell^{1}\right)$ have a weak derivative such that $|\nabla(f)|_{2} \in$ $W\left(L^{\infty}, \ell^{1}\right)$. Let $\delta, M>0$. Then,

$$
O_{\delta, M}(f) \leq \delta\lceil 1+2 \delta\rceil^{d} M\|\nabla(f)\|_{W\left(L^{\infty}, \ell^{1}\right)} .
$$

Proof. Let $Z \in \Delta_{M}$ and $Z^{\prime} \in \Delta$ such that $d_{\infty}\left(Z, Z^{\prime}\right) \leq \delta$. For each $k \in \Lambda$ let us call $B_{k}$ the ball of diameter $\delta$ centered in the middle point between $z_{k}$ and $z_{k}^{\prime}$. Then by the lemma,

$$
\left|f\left(z_{k}\right)-f\left(z_{k}^{\prime}\right)\right| \leq \sup _{B_{k}} \operatorname{ess}\|\nabla(f)\|_{2}\left|z_{k}-z_{k}^{\prime}\right|_{2} .
$$

Let $\sigma: \Lambda \rightarrow \mathbb{N}$ be a bijection. Given $\varepsilon>0$, there exists $c_{k} \in B_{k}$ such that

$$
\sup _{B_{k}} \operatorname{ess}\|\nabla(f)\|_{2} \leq\left\|\nabla(f)\left(c_{k}\right)\right\|_{2}+\frac{\varepsilon}{2^{\sigma(k)}} .
$$

Since $\left|z_{k}-z_{k}^{\prime}\right|_{2} \leq \delta$,

$$
\begin{aligned}
\left|f\left(z_{k}\right)-f\left(z_{k}^{\prime}\right)\right| & \leq\left(\left\|\nabla(f)\left(c_{k}\right)\right\|_{2}+\frac{\varepsilon}{2^{\sigma(k)}}\right) \delta \\
\left|z_{k}-c_{k}\right|_{2} & \leq \delta .
\end{aligned}
$$

Let us observe that,

$$
\sum_{k \in \Lambda} \frac{1}{2^{\sigma(k)}}=\sum_{k \in \mathbb{N}} \frac{1}{2^{k}}=1 .
$$

Moreover, by Observation 1.1,

$$
\operatorname{rel}_{\frac{1}{2}}\left(\left(c_{k}\right)_{k \in \Lambda}\right) \leq \operatorname{rel}_{\frac{1}{2}+\delta}(Z) \leq\lceil 1+2 \delta\rceil^{d} M .
$$

Now we simply estimate,

$$
\begin{aligned}
\sum_{k \in \Lambda}\left|f\left(z_{k}\right)-f\left(z_{k}^{\prime}\right)\right| & \leq \sum_{k \in \Lambda}\left(\left\|\nabla(f)\left(c_{k}\right)\right\|_{2}+\frac{\varepsilon}{2^{\sigma(k)}}\right) \delta \\
& \leq \delta\left(\sum_{k \in \Lambda}\left\|\nabla(f)\left(c_{k}\right)\right\|_{2}+\varepsilon\right) \\
& \leq \delta\left(\sum_{j \in \mathbb{Z}^{d}} \sum_{c_{k} \in[0,1)^{d}+j}\left\|\nabla(f)\left(c_{k}\right)\right\|_{2}+\varepsilon\right) \\
& \leq \delta\left(\sum_{j \in \mathbb{Z}^{d}} \sup _{0,1]^{d}+j}\|\nabla(f)\|_{2} \operatorname{rel}_{\frac{1}{2}}\left(\left(c_{k}\right)_{k \in \Lambda}\right)+\varepsilon\right) \\
& \leq \delta\lceil 1+2 \delta]^{d} M\|\nabla(f)\|_{W\left(L^{\infty}, \ell^{1}\right)}+\delta \varepsilon .
\end{aligned}
$$

The lemma follows by first letting $\varepsilon \rightarrow 0^{+}$and then taking supremum over all sets $Z$ and $Z^{\prime}$.

Lemma 2.9. Let $f \in W\left(C_{0}, \ell^{1}\right)$ have a weak derivative such that $|\nabla(f)|_{2} \in$ $W\left(L^{q}, \ell^{1}\right)$ and $d<q<\infty$. Let $\delta, M>0$. Then,

$$
O_{\delta, M}(f) \leq C \delta^{1-\frac{d}{q}}(1+2\lceil\delta / 2\rceil)^{2 d} M\|\nabla(f)\|_{W\left(L^{q}, \ell^{1}\right)} .
$$

where the constant $C$ depends only on $q$ and $d$. 
Proof. Let $Z \in \Delta_{M}$ and $Z^{\prime} \in \Delta$ such that $d_{\infty}\left(Z, Z^{\prime}\right) \leq \delta$. For each $k \in \Lambda$ let $c_{k}$ be the middle point between $z_{k}$ and $z_{k}^{\prime}$ and let $B_{k}$ be the closed ball centered in $c_{k}$ with diameter $\delta$. Let us call $n:=\lceil\delta / 2\rceil, Q:=[0,1)^{d}$ and $Q^{\prime}:=Q+\left[-\frac{\delta}{2}, \frac{\delta}{2}\right]^{d}$.

Morrey's inequality [8, pg. 143] guarantees the existence of a constant $C$ that depends only on $q$ and $d$ such that

$$
\left|f\left(z_{k}\right)-f\left(z_{k}^{\prime}\right)\right| \leq C \delta^{1-\frac{d}{q}}\|\nabla(f)\|_{L^{q}\left(B_{k}\right)} .
$$

Note that $d_{\infty}\left(\left(c_{k}\right)_{k}, Z\right) \leq \frac{\delta}{2}$ and therefore, according to observation 1.1,

$$
\operatorname{rel}_{\frac{1}{2}}\left(\left(c_{k}\right)_{k}\right) \leq \operatorname{rel}_{\frac{1}{2}+\frac{\delta}{2}}(Z) \leq(1+2 n)^{d} M .
$$

Now let us estimate,

$$
\begin{aligned}
\sum_{k \in \Lambda}\left|f\left(z_{k}\right)-f\left(z_{k}^{\prime}\right)\right| \leq & C \delta^{1-\frac{d}{q}} \sum_{k \in \Lambda}\|\nabla(f)\|_{L^{q}\left(B_{k}\right)} \\
\leq & C \delta^{1-\frac{d}{q}} \sum_{j \in \mathbb{Z}^{d}} \sum_{c_{k} \in Q+j}\|\nabla(f)\|_{L^{q}\left(B_{k}\right)} \\
\leq & C \delta^{1-\frac{d}{q}} \sum_{j \in \mathbb{Z}^{d}} \operatorname{rel}_{\frac{1}{2}}\left(\left(c_{k}\right)_{k}\right)\|\nabla(f)\|_{L^{q}\left(Q^{\prime}+j\right)} \\
& \text { since }\left|c_{k}-z_{k}\right|_{2}<\frac{\delta}{2}, B_{k} \subseteq Q^{\prime}+j . \\
\leq & C \delta^{1-\frac{d}{q}}(1+2 n)^{d} M \sum_{j \in \mathbb{Z}^{d}}\|\nabla(f)\|_{L^{q}\left(Q^{\prime}+j\right)} .
\end{aligned}
$$

Since $Q^{\prime} \subset \bigcup_{l \in([-n, n] \cap \mathbb{Z})^{d}} Q+l$, Minkowski's inequality implies that

$$
\|\nabla(f)\|_{L^{q}\left(Q^{\prime}+j\right)} \leq \sum_{l \in([-n, n] \cap \mathbb{Z})^{d}}\|\nabla(f)\|_{L^{q}(Q+j+l)} .
$$

Hence,

as desired.

$$
\begin{aligned}
\sum_{k \in \Lambda}\left|f\left(z_{k}\right)-f\left(z_{k}^{\prime}\right)\right| & \leq C \delta^{1-\frac{d}{q}}(1+2 n)^{d} M \sum_{j \in \mathbb{Z}^{d}} \sum_{l \in([-n, n] \cap \mathbb{Z})^{d}}\|\nabla(f)\|_{L^{q}(Q+j+l)} \\
& =C \delta^{1-\frac{d}{q}}(1+2 n)^{d} M \sum_{l \in([-n, n] \cap \mathbb{Z})^{d}} \sum_{j \in \mathbb{Z}^{d}}\|\nabla(f)\|_{L^{q}(Q+j+l)} \\
& =C \delta^{1-\frac{d}{q}}(1+2 n)^{2 d} M\|\nabla f\|_{W\left(L^{q}, \ell^{1}\right)} .
\end{aligned}
$$

Let us now prove Theorems 2.5 and 2.6.

Proof. Let us first prove Theorem 2.5. Suppose that $\delta:=d_{\infty}(Y, Z)<L$. We will show that $Z$ is a sampling set. This will prove that $\rho(Y) \geq L$.

Using Theorem 2.3 and Lemma 2.8 (and ignoring the trivial case $\varphi \equiv 0$ ),

$$
\begin{aligned}
\left\|\Upsilon_{Y}-\Upsilon_{Z}\right\|_{p} & \leq O_{\delta, M}(\varphi)^{\frac{1}{p}} O_{\delta, N}(\varphi)^{\frac{1}{p^{\prime}}} \\
& \leq \delta\lceil 1+2 \delta\rceil^{d} M^{\frac{1}{p}} N^{\frac{1}{p^{\prime}}}\|\nabla \varphi\|_{W\left(L^{\infty}, \ell^{1}\right)} \\
& <L\lceil 1+2 L\rceil^{d} M^{\frac{1}{p}} N^{\frac{1}{p^{\prime}}}\|\nabla \varphi\|_{W\left(L^{\infty}, \ell^{1}\right)} \leq A .
\end{aligned}
$$

Hence, if $A^{\prime}:=A-\left\|\Upsilon_{Y}-\Upsilon_{Z}\right\|>0$, for $c \in l^{2}(\Lambda)$,

$$
\left\|\Upsilon_{Z}(c)\right\| \geq\left\|\Upsilon_{Y}(c)\right\|-\left\|\left(\Upsilon_{Y}-\Upsilon_{Z}\right)(c)\right\| \geq A\|c\|-\left\|\Upsilon_{Y}-\Upsilon_{Z}\right\|\|c\|=A^{\prime}\|c\|
$$


Hence, according to proposition $1.2, Z$ is a set of sampling.

Theorem 2.6, follows by the same argument but applying Lemma 2.9 (instead of Lemma 2.8).

To end this subsection, we show that our estimates are somehow biased by the arbitrary windowing of functions. In a very simple example we show how the estimates on $\rho$ can be improved by taking into account the support of $\varphi$.

Example 2.1. Let $\varphi: \mathbb{R} \rightarrow \mathbb{R}$ be defined by $\varphi(x):=(1-|x|) \chi_{[-1,1]}(x)$ and $\Lambda=\mathbb{Z}$. Then $\mathcal{S}^{p}$ is the space of continuous linear splines with integer nodes that belong to $L^{p}$. It is easily seen that the general assumptions of this section are satisfied.

Let $Y:=\mathbb{Z}$. Then $Y$ is a sampling set for $\mathcal{S}^{p}$ - in fact, it is an interpolation set. With the notation of Theorem 2.5, $\Upsilon_{Y}=I d, A=1, N=M=1$ and $\left\|\varphi^{\prime}\right\|_{W\left(L^{\infty}, \ell^{1}\right)}=2$; so the estimate given by Theorem 2.5 is $\rho(Y) \geq 1 / 4$.

However, if in the proof of Theorem 2.5, we take into account the fact that whenever $d_{\infty}\left(\left\{z_{k}\right\}_{k}, Y\right)<1 / 2$, for each $k \in \mathbb{Z}$, the generator function $\varphi$ vanishes in one of the points $z_{k}-(k-1)$ or $z_{k}-(k+1)$, we obtain the sharper estimate $\rho(Y) \geq 1 / 3$.

2.3. Optimal perturbations. In this section we prove that, given a sampling set $Y$ there exist optimal local perturbations. More precisely, given $\delta$, there is a way of moving each point of $Y$ in less than $\delta$ so that the lower bound of the corresponding sampling operator attains its maximum among all such perturbations.

Theorem 2.10. Let $Y \equiv\left\{y_{k}\right\}_{k \in \Lambda}$ be a (relatively separated) sampling set for $\mathcal{S}^{p}$

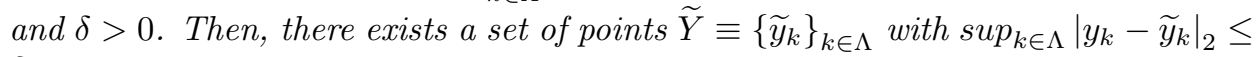
$\delta$ such that

$$
\zeta(\widetilde{Y})=\sup \left\{\zeta(Z): Z \equiv\left\{z_{k}\right\}_{k \in \Lambda}, \sup _{k \in \Lambda}\left|y_{k}-z_{k}\right|_{2} \leq \delta\right\},
$$

where $\zeta(Z)$ denotes the lower bound of the corresponding sampling operator $\Upsilon_{Z}$.

The main tool to establish Theorem 2.10 will be the map $\Upsilon$ from the previous subsections, but this time considered with coarser topologies in both the domain and codomain.

For $B\left(\ell^{p}(\Lambda)\right)$ we consider the strong operator topology (SOT), which is the initial topology induced by the evaluations, that is, the topology of pointwise convergence (in norm $p$ ).

We will define in $\Delta$ a weak topology that will allow us to prove Theorem 2.10 by continuity and compactness arguments.

Definition 2.4. Given $Y \in \Delta$ and $r>0$, we define $E(Y, r)$ as the ball of center $Y$ and radius $\delta$,

$$
E(Y, r):=B_{\delta}(Y)=\left\{Z \in \Delta: d_{\infty}(Z, Y) \leq \delta\right\}
$$

considered as a subspace of $\prod_{k \in \Lambda} B\left(y_{k}, \delta\right)$, this latter space having the product topology. Here $B\left(y_{k}, \delta\right) \subseteq \mathbb{R}^{d}$ is the closed ball of center $y_{k}$ and radius $\delta$ in norm 2. Consequently, $E(Y, r)$ has the pointwise convergence topology.

Observation 2.1. According to Observation 1.1, $E(Y, r)$ is the set of all $\Lambda$-indexed sets that are at a uniform distance smaller than $\delta$ to $Y$ (because these are necessarily relatively separated.) Hence, $E(Y, r)=\prod_{k \in \Lambda} B\left(y_{k}, \delta\right)$. 
Since the product is countable, $E(Y, r)$ is metrizable (although, of course, $d_{\infty}$ is not a metric for this topology.) Moreover, by Tychonoff's Theorem, E $(Y, r)$ is compact.

Definition 2.5. We define the weak topology in $\Delta$ as the final topology of the family of inclusion maps $\left\{\iota_{Y, r}: E(Y, r) \hookrightarrow \Delta: Y \in \Delta, r>0\right\}$. That is, the finest topology that makes each of these inclusions continuous. Hereafter, $(\Delta, w)$ will denote the set $\Delta$ considered with its weak topology.

The weak topology in $\Delta$ is characterized by the following universal property: a function $f:(\Delta, w) \rightarrow V$, from $\Delta$ to a topological space $V$, is continuous if and only if, for every $Y \in \Delta$ and $r>0$, the function $f_{Y, r}:=f \circ \iota_{Y, r}$ is continuous.

Note that the weak topology is finer than the product topology on $\Delta$, which is the pointwise convergence topology. The above defined weak topology can be regarded as a 'dominated convergence' topology since convergence in the basic neighborhoods $E(Y, r)$ means pointwise convergence plus the existence of some sort of uniform dominant set $Y$, although we do not impose a summability condition on it.

We first study the weak continuity of the functions induced by the amalgam $W\left(C_{0}, \ell^{1}\right)$.

Lemma 2.11. Let $f \in W\left(C_{0}, \ell^{1}\right)$. Then, the induced function

$$
f:(\Delta, w) \rightarrow l^{1}(\Lambda), \quad f(Z)=\left(f\left(z_{k}\right)\right)_{k \in \Lambda}
$$

is continuous.

The fact that this function is well-defined was observed in the lemmas before Theorem 2.2.

Proof. According to the universal property it suffices to check that, given $Y \in \Delta$ and $r>0$ the function $f_{Y, r}:=f \circ \iota_{Y, r}: E(Y, r) \rightarrow l^{1}(\Lambda)$ is continuous.

Since $E(Y, r)$ is metrizable, it will suffice to check that if $\left\{Z^{n}\right\}_{n \in \mathbb{N}} \subseteq E(Y, r)$ and $Z \in E(Y, r)$ are such that $Z^{n} \longrightarrow_{n} Z$ then, $f_{Y, r}\left(Z^{n}\right)=f\left(Z^{n}\right) \stackrel{l^{1}}{\longrightarrow}{ }_{n} f(Z)=f_{Y, r}(Z)$.

Say, $Z \equiv\left\{z_{k}\right\}_{k \in \Lambda}, Y \equiv\left\{y_{k}\right\}_{k \in \Lambda}$ and $Z^{n} \equiv\left\{z_{k}^{n}\right\}_{k \in \Lambda}$.

Let us call $I:=[0,1]^{d}, I^{\prime}:=I+[-r, r]^{d}$ and $N:=\lceil r\rceil$. Observe that

$$
I^{\prime} \subseteq I+[-N, N]^{d} \subseteq \bigcup_{k \in([-N, N] \cap \mathbb{Z})^{d}} I+k .
$$

Let us estimate,

$$
\begin{aligned}
\sum_{j \in \mathbb{Z}^{d}} \sup _{I^{\prime}+j}|f| & \leq \sum_{j \in \mathbb{Z}^{d}} \sup _{I+j+[-N, N]^{d}}|f| \\
& \leq \sum_{j \in \mathbb{Z}^{d}} \sum_{k \in([-N, N] \cap \mathbb{Z})^{d}} \sup _{I+j+k}|f| \\
& \leq \sum_{k \in([-N, N] \cap \mathbb{Z})^{d}} \sum_{j \in \mathbb{Z}^{d}} \sup _{I+j+k}|f| \\
& =(2 N+1)^{d}\|f\|_{W\left(C_{0}, \ell^{1}\right)}<\infty .
\end{aligned}
$$

So, given $\varepsilon>0$ there exists a cube $Q$ such that

$$
\sum_{k \in \mathbb{Z}^{d} \backslash Q} \sup _{I^{\prime}+k}|f|<\frac{\varepsilon}{4 \overline{\operatorname{rel}_{\frac{1}{2}}}(Y)} .
$$


Since $Y$ is relatively separated, only a finite number of its elements lie in $Q+I$. Since $z_{k}^{n} \rightarrow{ }_{n} z_{k}$ for each $k$, there exists $n_{0} \in \mathbb{N}$ such that for $n \geq n_{0}$,

$$
\sum_{k: y_{k} \in Q+I}\left|f\left(z_{k}^{n}\right)-f\left(z_{k}\right)\right|<\frac{\varepsilon}{2} .
$$

If $n \geq n_{0}$,

$$
\begin{aligned}
\sum_{k \in \Lambda}\left|f\left(z_{k}^{n}\right)-f\left(z_{k}\right)\right| & =\sum_{k: y_{k} \in Q+I}\left|f\left(z_{k}^{n}\right)-f\left(z_{k}\right)\right|+\sum_{k: y_{k} \notin Q+I}\left|f\left(z_{k}^{n}\right)-f\left(z_{k}\right)\right| \\
& \leq \frac{\varepsilon}{2}+\sum_{k: y_{k} \notin Q+I}\left|f\left(z_{k}^{n}\right)-f\left(z_{k}\right)\right| \\
& \leq \frac{\varepsilon}{2}+\sum_{j \in \mathbb{Z}^{d}} \sum_{y_{k} \in(I+j) \backslash Q+I}\left|f\left(z_{k}^{n}\right)-f\left(z_{k}\right)\right| \\
& =\frac{\varepsilon}{2}+\sum_{j \in \mathbb{Z}^{d} \backslash Q} \sum_{y_{k} \in(I+j) \backslash Q+I}\left|f\left(z_{k}^{n}\right)-f\left(z_{k}\right)\right| \\
& \leq \frac{\varepsilon}{2}+\sum_{j \in \mathbb{Z}^{d} \backslash Q} \overline{\operatorname{rel}_{\frac{1}{2}}}(Y) 2 \underset{\sup ^{\prime}+j}{\sup }|f| \\
& \leq \frac{\varepsilon}{2}+\frac{\varepsilon}{2}=\varepsilon .
\end{aligned}
$$

Hence, if $n \geq n_{o},\left\|f(Z)-f\left(Z^{n}\right)\right\|_{1} \leq \varepsilon$.

Now we can state the main result towards the proof of Theorem 2.10.

Theorem 2.12. The map

$$
\Upsilon:(\Delta, w) \rightarrow\left(B\left(\ell^{p}(\Lambda)\right), S O T\right)
$$

that maps a set $Z$ to its corresponding sampling operator $\Upsilon_{Z}$ is continuous.

Proof. It is enough to check that given $Y \in \Delta$ and $r>0$ the function $f_{Y, r}:=$ $f \circ \iota_{Y, r}: E(Y, r) \rightarrow l^{1}(\Lambda)$ is continuous.

Using Observation 1.1, for each $Z \in E(Y, r)$,

$$
\operatorname{rel}_{\frac{1}{2}}(Z) \leq \operatorname{rel}_{\frac{1}{2}+r}(Y) .
$$

Since $E(Y, r)$ is metrizable, let us show that if $\left\{Z^{n}\right\}_{n \in \mathbb{N}} \subseteq E(Y, r)$ and $Z \in$ $E(Y, r)$ are such that $Z^{n} \longrightarrow_{n} Z$, then $\Upsilon\left(Z^{n}\right) \stackrel{S O T}{\longrightarrow} \Upsilon_{Z}$.

Given $c \equiv\left(c_{k}\right)_{k \in \Lambda} \in \ell^{p}(\Lambda)$ we will show that $\Upsilon\left(Z^{n}\right)(c) \stackrel{\ell^{p}}{\longrightarrow} \Upsilon_{Z}(c)$. Let $\varepsilon>0$ be arbitrary.

For each $W \in \Delta$,

$$
\begin{aligned}
\sum_{k \in \Lambda}\left|\varphi\left(w_{k}\right)\right| & =\sum_{j \in \mathbb{Z}^{d}} \sum_{w_{k} \in[0,1)^{d}+j}\left|\varphi\left(w_{k}\right)\right| \\
& \leq \operatorname{rel}_{\frac{1}{2}}(W)\|\varphi\|_{W\left(C_{0}, \ell^{1}\right)} .
\end{aligned}
$$


Then, for each $W \in E(Y, r)$ and $k \in \Lambda$,

$$
\begin{aligned}
\sum_{l \in \Lambda}\left|\varphi\left(w_{l}-x_{k}\right)\right| & \leq \operatorname{rel}_{\frac{1}{2}}\left(W-x_{k}\right)\|\varphi\|_{W\left(C_{0}, \ell^{1}\right)} \\
& =\operatorname{rel}_{\frac{1}{2}}(W)\|\varphi\|_{W\left(C_{0}, \ell^{1}\right)} \\
& \leq \operatorname{rel}_{\frac{1}{2}+r}(Y)\|\varphi\|_{W\left(C_{0}, \ell^{1}\right)} .
\end{aligned}
$$

Similarly, since $X$ is relatively separated, for $l \in \Lambda$,

$$
\begin{aligned}
\sum_{k \in \Lambda}\left|\varphi\left(w_{l}-x_{k}\right)\right| & \leq \operatorname{rel}_{\frac{1}{2}}\left(w_{l}-X\right)\|\varphi\|_{W\left(C_{0}, \ell^{1}\right)} \\
& \leq \operatorname{rel}_{\frac{1}{2}}(X)\|\varphi\|_{W\left(C_{0}, \ell^{1}\right)} .
\end{aligned}
$$

Hence, if we set $M:=\max \left\{\operatorname{rel}_{\frac{1}{2}+r}(Y), \operatorname{rel}_{\frac{1}{2}}(X)\right\}\|\varphi\|_{W\left(C_{0}, \ell^{1}\right)}$ the following uniform bounds hold:

$$
\begin{aligned}
& \sum_{j \in \Lambda}\left|\varphi\left(z_{j}^{n}-x_{k}\right)\right|, \sum_{j \in \Lambda}\left|\varphi\left(z_{j}-x_{k}\right)\right| \leq M, \text { for every } k \in \Lambda \text { and } n \in \mathbb{N}, \\
& \sum_{k \in \Lambda}\left|\varphi\left(z_{j}^{n}-x_{k}\right)\right|, \sum_{k \in \Lambda}\left|\varphi\left(z_{j}-x_{k}\right)\right| \leq M, \text { for every } j \in \Lambda \text { and } n \in \mathbb{N} .
\end{aligned}
$$

Let us first assume that $p>1$. Since $c \in \ell^{p}(\Lambda)$, there exists a finite set $\Lambda_{0} \subseteq \Lambda$ such that,

$$
\sum_{k \in \Lambda \backslash \Lambda_{0}}\left|c_{k}\right|^{p}<\frac{\varepsilon}{2 M^{\frac{p}{p^{\prime}}+1}} .
$$

Let us estimate the variation of the sampling operator like we did before,

$$
\begin{aligned}
\| \Upsilon( & \left.Z^{n}\right)(c)-\Upsilon_{Z}(c) \|_{p}^{p}=\sum_{j \in \Lambda}\left|\sum_{k \in \Lambda} c_{k}\left(\varphi\left(z_{j}^{n}-x_{k}\right)-\varphi\left(z_{j}-x_{k}\right)\right)\right|^{p} \\
& \leq \sum_{j \in \Lambda}\left(\sum_{k \in \Lambda}\left|c_{k}\right|\left|\varphi\left(z_{j}^{n}-x_{k}\right)-\varphi\left(z_{j}-x_{k}\right)\right|^{\frac{1}{p}}\left|\varphi\left(z_{j}^{n}-x_{k}\right)-\varphi\left(z_{j}-x_{k}\right)\right|^{\frac{1}{p^{\prime}}}\right)^{p} \\
& \leq \sum_{j \in \Lambda} \sum_{k \in \Lambda}\left|c_{k}\right|^{p}\left|\varphi\left(z_{j}^{n}-x_{k}\right)-\varphi\left(z_{j}-x_{k}\right)\right|\left(\sum_{k \in \Lambda}\left|\varphi\left(z_{j}^{n}-x_{k}\right)-\varphi\left(z_{j}-x_{k}\right)\right|\right)^{\frac{p}{p^{\prime}}} \\
& \leq M^{\frac{p}{p^{\prime}}} \sum_{j \in \Lambda} \sum_{k \in \Lambda}\left|c_{k}\right|^{p}\left|\varphi\left(z_{j}^{n}-x_{k}\right)-\varphi\left(z_{j}-x_{k}\right)\right| \\
& \leq M^{\frac{p}{p^{\prime}}} \sum_{k \in \Lambda}\left|c_{k}\right|^{p} \sum_{j \in \Lambda}\left|\varphi\left(z_{j}^{n}-x_{k}\right)-\varphi\left(z_{j}-x_{k}\right)\right| \\
& \leq M^{\frac{p}{p^{\prime}}}\left(\sum_{k \in \Lambda_{0}}\left|c_{k}\right|^{p} \sum_{j \in \Lambda}\left|\varphi\left(z_{j}^{n}-x_{k}\right)-\varphi\left(z_{j}-x_{k}\right)\right|\right. \\
& \leq M^{\frac{p}{p^{\prime}}}\left(\sum_{k \in \Lambda_{0}}\left|c_{k}\right|^{p} \sum_{j \in \Lambda}\left|\varphi\left(z_{j}^{n}-x_{k}\right)-\varphi\left(z_{j}-x_{k}\right)\right|+M \sum_{k \in \Lambda \backslash \Lambda_{0}}\left|\sum_{k}\right|^{p}\right) \\
& \left.\sum_{k \in \Lambda_{0}}\right|^{p} \sum_{j \in \Lambda} \mid \varphi\left(z_{j}^{n}-\left.x_{k}\right|^{p} \sum_{j \in \Lambda}\left|\varphi\left(z_{j}^{n}-x_{k}\right)-\varphi\left(z_{j}-x_{k}\right)\right|\right) \\
& \left.+z_{j}-x_{k}\right) \mid+\frac{\varepsilon}{2} .
\end{aligned}
$$


When $p=1$ a similar (simpler) calculation establishes the same inequality (in this case $\frac{p}{p^{\prime}}=0$.) We claim that for each $k \in \Lambda, Z^{n}-x_{k} \stackrel{w}{\rightarrow}_{n} Z-x_{k}$. Since $Z^{n}, Z \in E(Y, r)$, we also have that $Z^{n}-x_{k}, Z-x_{k} \in E\left(Y-x_{k}, r\right)$. Since for each $j, z_{j}^{n} \rightarrow_{n} z_{j}$, it turns out that $z_{j}^{n}-x_{k} \rightarrow_{n} z_{j}-x_{k}$. Then $Z^{n}-x_{k} \rightarrow_{n} Z-x_{k}$ in $E\left(Y-x_{k}, r\right)$ (product topology). Since the inclusion map $E\left(Y-x_{k}, r\right) \hookrightarrow(\Delta, w)$ is continuous, $Z^{n}-x_{k} \rightarrow_{n} Z-x_{k}$ in $(\Delta, w)$ and the claim follows.

According to Lemma 2.11, for each $k \in \Lambda_{0}$,

$$
\sum_{j \in \Lambda}\left|\varphi\left(z_{j}^{n}-x_{k}\right)-\varphi\left(z_{j}-x_{k}\right)\right| \underset{n}{\rightarrow} 0 .
$$

Since $\Lambda_{0}$ is finite, it follows that there exists $n_{0} \in \mathbb{N}$ such that if $n \geq n_{0}$

$$
M^{\frac{p}{p^{\prime}}} \sum_{k \in \Lambda_{0}}\left|c_{k}\right|^{p} \sum_{j \in \Lambda}\left|\varphi\left(z_{j}^{n}-x_{k}\right)-\varphi\left(z_{j}-x_{k}\right)\right|<\frac{\varepsilon}{2} .
$$

Hence, for $n \geq n_{0},\left\|\Upsilon\left(Z^{n}\right)(c)-\Upsilon_{Z}(c)\right\|_{p}^{p}<\varepsilon$.

Lemma 2.13. The map

$$
\zeta:\left(B\left(\ell^{p}(\Lambda)\right), S O T\right) \rightarrow \mathbb{R}, \zeta(T)=\inf _{\|c\|_{p}=1}\|T(c)\|_{p}
$$

is upper-semicontinuous. (i.e. $\zeta^{-1}((-\infty, \alpha))$ is open for each $\left.\alpha \in \mathbb{R}\right)$

Proof. Given $\alpha \in \mathbb{R}$ it suffices to show that the complement of $\zeta^{-1}((-\infty, \alpha))$ is closed in the strong topology. For each $c \in \ell^{p}$ let us call $e v_{c}$ the evaluation map in $c$, i.e. $e v_{c}(T)=T(c)$.

The lower bound of an operator is greater than or equal to $\alpha$ if and only if it maps each $c \in \ell^{p}$ of norm 1 out of the open ball of radius $\alpha$. That is,

$$
\zeta^{-1}([\alpha, \infty))=\bigcap_{\|c\|_{p}=1} e v_{c}^{-1}\left(\ell^{p} \backslash B_{\alpha}^{\circ}(0)\right) .
$$

For each $c \in \ell^{p}$ of norm 1 , since $e v_{c}$ is continuous with the strong topology, and $\ell^{p} \backslash B_{\alpha}^{0}(0)$ is closed in $\ell^{p}$, it turns out that $e v_{c}^{-1}\left(\ell^{p} \backslash B_{\alpha}^{0}(0)\right)$ is SOT-closed. The arbitrary intersection of closed sets is closed.

We can finally prove Theorem 2.10 .

Theorem. Let $Y \equiv\left\{y_{k}\right\}_{k \in \Lambda}$ be a (relatively separated) sampling set for $\mathcal{S}^{p}$ and $\delta>0$. Then, there exists a set of points $\widetilde{Y} \equiv\left\{\widetilde{y}_{k}\right\}_{k \in \Lambda}$ with sup $p_{k \in \Lambda}\left|y_{k}-\widetilde{y}_{k}\right|_{2} \leq \delta$ such that

$$
\zeta(\tilde{Y})=\sup \left\{\zeta(Z): Z \equiv\left\{z_{k}\right\}_{k \in \Lambda}, \sup _{k \in \Lambda}\left|y_{k}-z_{k}\right|_{2} \leq \delta\right\},
$$

where $\zeta(Z)$ denotes the lower bound of the corresponding sampling operator $\Upsilon_{Z}$.

Proof. Composing the maps of both lemmas, we see that the map $\zeta: \Delta \rightarrow \mathbb{R}$ that sends a set of points to the lower bound of its sampling operator is upper semicontinuous. If we further compose this map with the inclusion $\iota_{Y, \delta}: E(Y, \delta) \hookrightarrow$ $\Delta$ we have an upper-semicontinuous map $E(Y, \delta) \rightarrow \mathbb{R}$ with compact domain that must therefore attain some maximum $\tilde{Y}$. 
Remark 2.2. The same argument shows that there exists a configuration of points $\tilde{Z}$ optimizing the lower bound of the operator $\left.f \mapsto\left(f\left(\tilde{z}_{k}\right)\right)_{k}\right)$. Indeed, since the Bessel map $c \mapsto \sum_{k} c_{k} \varphi(\cdot-k)$ is a norm-continuous isomorphism between $\ell^{p}$ and $\mathcal{S}^{p}$, it induces a SOT isomorphism between $B\left(\ell^{p}, \ell^{p}\right)$ and $B\left(\mathcal{S}^{p}, \ell^{p}\right)$. Composing the map of the proof above with this isomorphism, it turns out that the map $(\Delta, w) \ni$ $\left.Z \mapsto\left(f \mapsto\left(f\left(z_{k}\right)\right)_{k}\right)\right) \in\left(B\left(\mathcal{S}^{p}, \ell^{p}\right), S O T\right)$ is continuous.

\section{Perturbation of Bases}

\section{General assumption for section 3 .}

Throughout this section let $\varphi \in W\left(C_{0}, \ell^{1}\right)$ and $1 \leq p<\infty$.

Given a relatively separated set of points $X \equiv\left\{x_{k}\right\}_{k \in \Lambda}$ we want to construct a spline-type space $\mathcal{S}^{p}$. We start by defining it as $\mathcal{S}^{p}=\mathcal{S}^{p}(\varphi, X)=\overline{\operatorname{span}\left(\left\{\varphi\left(\cdot-x_{k}\right)\right\}_{k \in \Lambda}\right)}$, where the closure is taken in $L^{p}$ norm. In order to have a spline-type space it remains to check whether the set of translates $\left\{\varphi\left(\cdot-x_{k}\right)\right\}_{k \in \Lambda}$ forms a p-Riesz sequence.

Definition 3.1. Let $X \equiv\left\{x_{k}\right\}_{k \in \Lambda}$ be a relatively separated set of points. If the set of translates $\left\{\varphi\left(\cdot-x_{k}\right)\right\}_{k \in \Lambda}$ forms a p-Riesz sequence we call $X$ a Riesz set (for $\varphi)$.

The bounds of the $p$-Riesz sequence $\left\{\varphi\left(\cdot-x_{k}\right)\right\}_{k \in \Lambda}$ will be called bounds of $X$.

When $p=2$ and the set of points is a lattice the condition of being a Riesz set is known to be equivalent to a certain condition on $\hat{\varphi}$.

Theorem 3.1. [7], [4] If $p=2$ and there exists constants $A, B>0$ such that for almost every $x \in[0,1]^{d}$

$$
A \leq \sum_{k \in \mathbb{Z}^{d}}|\hat{\varphi}(x+j)|^{2} \leq B
$$

then the set $\mathbb{Z}^{d}$ is a Riesz set for $\varphi$ with bounds $A$ and $B$.

Clearly this results extends to general lattices other than $\mathbb{Z}^{d}$.

Remark 3.1. If $\varphi \in W\left(C_{0}, \ell^{1}\right)$, it can be shown by means of the Poisson summation formula that the periodization in (5) is a continuous function. Thus the condition in (5) is equivalent to $\sum_{k \in \mathbb{Z}^{d}}|\hat{\varphi}(x+j)|^{2} \neq 0$, for every $x \in[0,1]^{d}$.

In this section we parallel the techniques of the last section to study the possibility of disturbing Riesz sets. This, combined with Theorem 3.1 will yield a criterion for a space generated by irregular translations to be a spline-type space.

Definition 3.2. Let $X \equiv\left\{x_{k}\right\}_{k \in \Lambda}$ be a Riesz set. Let $\tau(X)$ be the supremum of the set of $\delta \geq 0$ such that every set of points $Y \equiv\left\{y_{k}\right\}_{k \in \Lambda}$ such that $\left|x_{k}-y_{k}\right|_{2} \leq \delta$, is a Riesz set. We call $\tau(X)$ the Riesz-radius of $X$.

We will show that the Riesz radius of a Riesz set is always positive.

Let us introduce the following notation.

Definition 3.3. If $X \equiv\left\{x_{k}\right\}_{k \in \Lambda}$ is a relatively separated set of points let us call $S_{X}$ the Bessel map of $\left\{\varphi\left(\cdot-x_{k}\right)\right\}_{k \in \Lambda}$. That is

$$
S_{X}: \ell^{p}(\Lambda) \longrightarrow L^{p}\left(\mathbb{R}^{d}\right), S_{X}(c)=\sum_{k \in \mathbb{Z}^{d}} c_{k} \varphi\left(\cdot-x_{k}\right) .
$$


It is easily shown that $S_{X}$ is well defined and bounded for every relatively separated set $X$. Moreover $X$ is a Riesz set if and only if $S_{X}$ is bounded below (cf. subsection 1.2).

Lemma 3.2. Let $M, \delta>0, X \in \Delta_{M}$ and $Y \in \Delta$ such that $d_{\infty}(X, Y) \leq \delta$. Then $\left\|S_{X}-S_{Y}\right\| \leq O_{\delta, M}(\varphi)^{\frac{1}{p^{\prime}}} O_{\delta, 1}(\varphi)^{\frac{1}{p}}$.

Proof. For $c \in \ell^{p}(\Lambda)$ let us estimate,

$$
\begin{aligned}
\| S_{X} & -S_{Y}(c) \|_{p}^{p}=\int_{\mathbb{R}^{d}}\left|\sum_{k \in \Lambda} c_{k}\left(\varphi\left(x-x_{k}\right)-\varphi\left(x-y_{k}\right)\right)\right|^{p} d x \\
& \leq \int_{\mathbb{R}^{d}}\left(\sum_{k \in \Lambda}\left|c_{k}\right|\left|\varphi\left(x-x_{k}\right)-\varphi\left(x-y_{k}\right)\right|^{\frac{1}{p}}\left|\varphi\left(x-x_{k}\right)-\varphi\left(x-y_{k}\right)\right|^{\frac{1}{p^{\prime}}}\right)^{p} d x \\
& \leq \int_{\mathbb{R}^{d}} \sum_{k \in \Lambda}\left|c_{k}\right|^{p}\left|\varphi\left(x-x_{k}\right)-\varphi\left(x-y_{k}\right)\right|\left(\sum_{k \in \Lambda}\left|\varphi\left(x-x_{k}\right)-\varphi\left(x-y_{k}\right)\right|\right)^{\frac{p}{p^{\prime}}} d x
\end{aligned}
$$

where we used Hölder for the last inequality. Now, since $\operatorname{rel}_{\frac{1}{2}}\left(\left(x-x_{k}\right)_{k}\right)=\operatorname{rel}_{\frac{1}{2}}(X) \leq$ $M$ and $\left.d_{\infty}\left(\left(x-x_{k}\right)_{k}\right),\left(x-y_{k}\right)_{k}\right)=d_{\infty}(X, Y) \leq \delta$ and on the other hand $\operatorname{rel}_{\frac{1}{2}}\left(\left(x+j-x_{k}\right)_{j}\right)=$ $\operatorname{rel}_{\frac{1}{2}}\left(\mathbb{Z}^{d}\right)=1$ and $\left.d_{\infty}\left(\left(x+j-x_{k}\right)_{j}\right),\left(x+j-y_{k}\right)_{j}\right)=\left|x_{k}-y_{k}\right| \leq \delta$, we obtain:

$$
\begin{aligned}
& \left\|S_{X}-S_{Y}(c)\right\|_{p}^{p} \\
& \quad \leq O_{\delta, M}(\varphi)^{\frac{p}{p^{\prime}}} \sum_{k \in \Lambda}\left|c_{k}\right|^{p} \int_{\mathbb{R}^{d}}\left|\varphi\left(x-x_{k}\right)-\varphi\left(x-y_{k}\right)\right| d x \\
& \quad \leq O_{\delta, M}(\varphi)^{\frac{p}{p^{\prime}}} \sum_{k \in \Lambda}\left|c_{k}\right|^{p} \int_{[0,1]^{d}} \sum_{j \in \mathbb{Z}^{d}}\left|\varphi\left(x+j-x_{k}\right)-\varphi\left(x+j-y_{k}\right)\right| d x \\
& \quad \leq O_{\delta, M}(\varphi)^{\frac{p}{p^{\prime}}} \sum_{k \in \Lambda}\left|c_{k}\right|^{p} \int_{[0,1]^{d}} O_{\delta, 1}(\varphi) d x \\
& \quad=O_{\delta, M}(\varphi)^{\frac{p}{p^{\prime}}} O_{\delta, 1}(\varphi)\|c\|_{p}^{p} .
\end{aligned}
$$

Hence, $\left\|S_{X}-S_{Y}\right\|_{p} \leq O_{\delta, M}(\varphi)^{\frac{1}{p^{\prime}}} O_{\delta, 1}(\varphi)^{\frac{1}{p}}$.

Theorem 3.3. The map

$$
\begin{aligned}
S:\left(\Delta, d_{\infty}^{*}\right) & \longrightarrow\left(B\left(\ell^{p}(\Lambda), L^{p}\left(\mathbb{R}^{d}\right)\right),\|\cdot\|\right) \\
X & \longmapsto S_{X}
\end{aligned}
$$

that maps a set of points to its Bessel operator is continuous.

Proof. For $X, Y \in \Delta$, if $\delta:=d_{\infty}(X, Y)<1$, by Lemmas 3.2 and 2.2,

$$
\left\|S_{X}-S_{Y}\right\| \leq O_{\delta, M}(\varphi)^{\frac{1}{p^{\prime}}} O_{\delta, 1}(\varphi)^{\frac{1}{p}} \rightarrow 0, \text { as } d_{\infty}^{*}(X, Y) \rightarrow 0 .
$$

Now we can prove the main result of the section.

Theorem 3.4. Let $X \equiv\left\{x_{k}\right\}_{k \in \Lambda}$ be a Riesz set. Then $\tau(X)>0$.

Hence if $0 \leq \delta<\tau(X)$ and $Y \equiv\left\{y_{k}\right\}_{k \in \Lambda}$ is a set of points such that $\left|x_{k}-y_{k}\right|_{2} \leq$ $\delta$, then $Y$ is a Riesz set. 
Proof. Since by Observation 1.1 every set that is uniformly close to $X$ is relatively separated it suffices to show that the subset $R:=\{Z \in \Delta: Z$ is a Riesz set $\}$ is open in $\left(\Delta, d_{\infty}^{*}\right)$.

Let $B B$ be the subset of $B\left(\ell^{p}(\Lambda), L^{p}\left(\mathbb{R}^{d}\right)\right)$ formed by those operators that are bounded below. As we observed before, $B B$ is open in the norm topology. Now, $R$ is the inverse image of $B B$ by the continuous application of Theorem 3.3. This completes the proof.

From this we derive the following criterion:

Theorem 3.5. Let $p=2$ and $\varphi$ have a weak derivative such that $|\nabla(\varphi)|_{2} \in$ $W\left(L^{\infty}, \ell^{1}\right)$. Suppose that $A, B, \delta>0$ satisfy:

- $A \leq \sum_{k \in \mathbb{Z}^{d}}|\hat{\varphi}(x+k)|^{2} \leq B$, for every $x \in[0,1]^{d}$,

- $\delta\lceil 1+2 \delta\rceil^{d}\|\nabla(\varphi)\|_{W\left(L^{\infty}, \ell^{1}\right)}<\sqrt{A}$.

Then, every set $X \equiv\left\{x_{k}\right\}_{k \in \mathbb{Z}^{d}}$ such that $\left|x_{k}-k\right|_{2} \leq \delta$, is a Riesz set for $\varphi$.

Proof. By Theorem 3.1, $\mathbb{Z}^{d}$ is a Riesz set with bounds $A$ and $B$. Let $X$ be a set such that $d_{\infty}\left(X, \mathbb{Z}^{d}\right) \leq \delta$. Using Lemma 3.2 and Lemma 2.8 we have,

$$
\left\|S_{X}-S\left(\mathbb{Z}^{d}\right)\right\| \leq O_{\delta, 1}(\varphi) \leq \delta\lceil 1+2 \delta\rceil^{d}\|\nabla(\varphi)\|_{W\left(L^{\infty}, \ell^{1}\right)}<\sqrt{A} .
$$

Since the operator $S\left(\mathbb{Z}^{d}\right)$ has lower bound $\sqrt{A}$, it follows that $S_{X}$ is bounded below. Hence, $X$ is also a Riesz set.

Remark 3.2. An analogous criterion for $|\nabla(\varphi)|_{2} \in W\left(L^{q}, \ell^{1}\right)$, and $q>d$ can be proved using Lemma 2.9. With the pertinent changes an arbitrary lattice can be used instead of $\mathbb{Z}^{d}$.

\section{Final Remarks}

Pointwise and convolution relations in amalgam spaces ([9]) imply that the Sampling and Bessel maps depend continuously on the generator function $\varphi$ in the $W\left(C_{0}, \ell^{1}\right)$ norm. This observation, combined with the results in this article, imply that both the sampling problem and the $p$-Riesz sequence of irregular translates condition are stable under small perturbations of the underlying points and the generator function (the former in $d_{\infty}$ metric and the latter in the $W\left(C_{0}, \ell^{1}\right)$ norm).

Stability results relying on amalgam space techniques have been used to strengthen sampling theorems; see for example [5] [1].

\section{Acknowledgements}

The initial research for this article was carried out during a visit of Ursula Molter at the Erwin Schroedinger Institute (special semester on Harmonic Analysis and Applications) and the University Vienna, thanks to an $\mathrm{Al} \beta$ an scholarship. We thank these institutions for their hospitality and support. We also benefited from conversations with C. Cabrelli and K. Gröchenig.

José Luis Romero acknowledges support from EUCETIFA to be able to complete the work at the University of Vienna, and thanks the NuHAG members for their hospitality during his stay. He holds a fellowship from the CONICET and thanks this institution for its support. 
The research of Ursula Molter and José Luis Romero is partially supported by Grants: PICT 15033, CONICET, PIP 5650, UBACyT X108.

The research of Hans G. Feichtinger is partially supported by the European Marie Curie Excellence Grant EUCETIFA (FP6-517154).

\section{REFERENCES}

[1] E. Acosta-Reyes, A. Aldroubi and I. Krishtal. On Stability of Sampling-Reconstruction Models, to appear in Special Issue on Mathematical Model for Image Processing, Adv. Comput. Math. (2008).

[2] A. Aldroubi and H. G. Feichtinger. Exact iterative reconstruction algorithm for multivariate irregularly sampled functions in spline-like spaces: The $L^{p}$-Theory. Proc. Amer. Math. Soc., 126(9) (1981) 2677-2686.

[3] A. Aldroubi and K. Gröchenig. Beurling-Landau-type theorems for non-uniform sampling in shift invariant spline spaces. J. Fourier Anal. Appl., 6(1) (2000) 93-103.

[4] A. Aldroubi and K. Gröchenig. Nonuniform sampling and reconstruction in shift-invariant spaces. SIAM Rev., 43(4) (2001) 585-620.

[5] A. Aldroubi and I. Krishtal. Robustness of sampling and reconstruction and Beurling-Landau-type theorems for shift invariant spaces. Appl. Comput. Harmon. Anal, 20(2) (2006) 250-260

[6] M. Bownik. The structure of shift-invariant subspaces of $L^{2}\left(\mathbf{R}^{n}\right)$. J. Funct. Anal., 177(2) (2000) 282-309.

[7] C. de Boor, R. A. DeVore, and A. Ron. The structure of finitely generated shift-invariant spaces in $L^{2}\left(\mathbb{R}^{d}\right)$. J. Funct. Anal., 119 (1) (1984) 37-78.

[8] L. Evans and R. Gariepy. Measure Theory and Fine Properties of Functions. CRC Press, 1992.

[9] H. G. Feichtinger. Banach convolution algebras of Wiener type. In Proc. Conf. on Functions, Series, Operators, Budapest 1980, volume 35 of Colloq. Math. Soc. Janos Bolyai. North-Holland, Amsterdam (1983) 509-524.

[10] H. G. Feichtinger. New results on regular and irregular sampling based on Wiener amalgams. In K. Jarosz, editor, Function spaces, Proc. Conf., Edwardsville/IL (USA) 1990, volume 136 of Lect. Notes Pure Appl. Math., New York, Marcel Dekker (1992) 107-121.

[11] H. G. Feichtinger and K. Gröchenig. Theory and practice of irregular sampling. In J. Benedetto and M. Frazier, editors, Wavelets: mathematics and applications, Studies in Advanced Mathematics, Boca Raton, FL, CRC Press (1994) 305-363.

[12] H. G. Feichtinger and T. Werther. Robustness of minimal norm interpolation in Sobolev algebras. In J.J.Benedetto and A.Zayed, editors, Sampling, Wavelets and Tomography. Birkhäuser Boston (2002) 8 3-113.

[13] C. Heil. An introduction to weighted Wiener amalgams. In M. Krishna, R. Radha, and S. Thangavelu, editors, Wavelets and their Applications (Chennai, January 2002), Allied Publishers, New Delhi (2003) 183-216.

[14] M. Ц̆. Kadec. The exact value of the Paley-Wiener constant. Dokl. Akad. Nauk SSSR, 155 (1964) 1253-1254.

[15] N. Levinson. On Non-Harmonic Fourier Series. Annals of Mathe. 37(4) (1936) 919-936.

[16] A. Ron and Z. Shen. Frames and stable bases for shift-invariant subspaces of $L^{2}\left(R^{d}\right)$. Canad. J. Math, 47(5) (1995) 1051-1094.

[17] J. Xian and S. Li. Sampling set conditions in weighted multiply generated shift-invariant spaces and their applications. Appl. Comput. Harmon. Anal., 23(2) (2007) 171-180. 
University Vienna, Faculty of Mathematics, Nordbergstrasse 15, Wien

E-mail address, Hans G. Feichtinger: hans.feichtinger@univie.ac.at

Departamento de Matemática, Facultad de Ciencias Exactas y Naturales, Universidad de Buenos Aires, Ciudad Universitaria, Pabellón I, 1428 Capital Federal,

ARGENTINA, AND CONICET, ARgEntina

E-mail address, Ursula M. Molter: umolter@dm.uba.ar

Departamento de Matemática, Facultad de Ciencias Exactas y Naturales, Universidad de Buenos Aires, Ciudad Universitaria, Pabellón I, 1428 Capital Federal,

ARGENTINA, AND CONICET, ARgEntina

E-mail address, José Luis Romero: jlromero@dm.uba.ar 